\title{
Dynamics of the boundary layer created by the explosion of a dense object in an ambient dilute gas triggered by a high power laser
}

\author{
Ryutaro Matsui, ${ }^{1,2}$ Yuji Fukuda, ${ }^{2, *}$ and Yasuaki Kishimoto ${ }^{1, \dagger}$ \\ ${ }^{1}$ Graduate School of Energy Science, Kyoto University, Gokasho, Uji, Kyoto 611-0011, Japan \\ ${ }^{2}$ Kansai Photon Science Institute (KPSI), National Institutes for Quantum and Radiological Science and Technology (QST), \\ 8-1-7 Umemidai, Kizugawa, Kyoto 619-0215, Japan
}

(Received 7 May 2019; published 8 July 2019)

\begin{abstract}
The dynamics of the boundary layer in between two distinct collisionless plasmas created by the interaction between a dense object modeling a cluster and a short laser pulse in the presence of an ambient gas is studied with two dimensional relativistic particle-in-cell simulations, which are found to be described by three successive processes. In the first phase, a collisionless electrostatic shock wave, launched near the cluster expansion front, reflects the ambient gas ions at a contact surface as a moving wall, which allows a particle acceleration with a narrower energy spread. In the second phase, the contact surface disappears and the compressed surface of the ambient gas ions passes over the shock potential, forming an overlapping region between the cluster expansion front and the compressed surface of the ambient gas. Here, another type of nonlinear wave is found to be evolved, leading to a relaxation of the shock structure, while continuing to reflect the ambient gas ions. The nonlinear wave exhibits a bipolar electric field structure that is sustained for a long timescale coupled with slowly evolving ion dynamics, suggesting that a quasistationary kinetic equilibrium dominated by electron vortices in the phase space is established. In the third phase, a rarefaction wave is triggered and evolves at the compressed surface of ambient gas. This is because some of the ambient gas ions tend to pass over the potential of the bipolar electric field. Simultaneously, a staircase structure, i.e., a kind of internal shock, is formed in the cluster due to the deceleration of cluster ions. Such structure formations and successive dynamics accompanied by the transitions from the shock wave phase through the overlapping phase to the rarefaction wave phase are considered to be a unique nature at the boundary layer created by an explosion of a dense plasma object in an ambient dilute plasma.
\end{abstract}

DOI: 10.1103/PhysRevE.100.013203

\section{INTRODUCTION}

Recently, high energy density plasmas produced by the interaction between high intensity lasers in the relativistic regime and various types of targets have generated much interest, since they exhibit prominent dynamics and structures when the parameters of laser and target are properly chosen, which leads to academic and also industrial applications [1,2]. A typical example is the generation of high energy particles aimed at a compact accelerator [3,4]. An interaction between a solid thin film on the order of micrometers and a high intensity laser has been studied to generate high energy ions by utilizing strong electrostatic sheath fields produced at the interface between the rear surface of target and vacuum [5-8]. Acceleration of ions by the shock induced in a near critical density plasma irradiated by a high intensity laser has been proposed, where the shock potential produced in the downstream region with high pressure reflects ions of that of the upstream across the interface between two plasmas [9-12].

These events are found to take place at the interface between plasma and vacuum and/or that between two

\footnotetext{
*fukuda.yuji@qst.go.jp

†kishimoto@energy.kyoto-u.ac.jp
}

plasmas with different characteristics, i.e., temperature, density, species, etc., which exhibits prominent dynamics and structures as a boundary layer [13-19]. Such boundary layers, which exhibit various phenomena including those mentioned above, emerge not only in the laboratory but also in space and the universe. The boundary layer produced by a supernova explosion in interstellar gas is a typical example, where complex spatiotemporal structures causing shock waves and particle accelerations are produced. In such events, two dimensional (2D) and/or three dimensional (3D) geometric effects, which correspond to cylindrical and/or spherical effects, play an important role in regulating the structures.

In order to elucidate the details of such structures, here we propose to utilize a cluster, i.e., a spherical dense object with a solid density, as a target [20-22]. This is because the cluster irradiated by a high power laser exhibits a nearly isotropic expansion, which is dimensionally similar to that of a spherical dense object in 3D space, e.g., the supernova explosion mentioned in the above.

The interaction of a cluster target with an intense laser pulse has been actively researched over the past two decades, because such a target allows an efficient coupling with the laser pulse compared to that of a solid, exhibiting prominent linear and nonlinear dynamics and associated optical properties [23-25]. Two types of dynamics are found to exist: one is the Coulomb explosion and the other is the ambipolar 
expansion [26]. The former takes place due to the Coulomb repulsive force when almost all cluster electrons are expelled from the ion core by laser fields. The latter takes place due to an ambipolar electric field when only peripheral electrons of the cluster are heated and expand. Meanwhile, these two types are not fully separated but incorporated and/or mixed depending on the parameters such as the size and the kind of material of the cluster, as discussed in Ref. [26]. Due to the variety of the interaction coupled with the collective effect of multiple clusters, which depends on the spatial distribution of clusters and the packing fraction, a wide range of application, both academically and industrially, has been proposed [27-31]. Recently, the internal degree of freedom of a hydrogen cluster causing converging shock has been utilized in achieving high energy protons over $200 \mathrm{MeV}$ [32].

In addition to such characteristics of clusters, the ambient gas surrounding them plays an important role in regulating the dynamics in the laser-irradiated cluster target [33-35]. As an example, the generation of a cylindrically symmetric blast wave [36-38], which is considered to be ascribed to the interaction between the cluster and the ambient gas, has been observed.

Here, we study the interaction between a high intensity laser and a cluster (carbon) in the presence of ambient gas (hydrogen) based on 2D particle-in-cell (PIC) simulations using EPIC3D (Extended Particle based Integrated Code) in the $(x, y)$ plane, where the cluster is modeled using a $2 \mathrm{D}$ circular structure. In a series of simulations, we find that the interaction is categorized into three successive processes characterized by the qualitatively different dynamics and structure.

In the first phase, a collisionless shock is launched and ambient gas ions are reflected by the shock potential by keeping the contact surface. In the second phase, the contact surface disappears and an overlapping region is formed while the ambient gas continues to be compressed, keeping a sharp boundary. A new type of nonlinear wave exhibiting a bipolar electric field is found to be formed around the compressed surface of the ambient gas and evolve. Ambient gas ions continue to be reflected by the potential keeping the shock structure. This suggests that a nonthermodynamic quasistationary kinetic equilibrium with the electron vortices in the phase space is established. In the third phase, the onset of a rarefaction wave takes place, which causes a destruction of the sharp boundary of the compressed ambient gas. Simultaneously, an internal-shock-like structure is formed in the cluster, leading to multiple ringlike and/or staircase structures in the cluster. Such ringlike structures are found to be widely observed after a supernova explosion [39].

Here, it is noted that present 2D simulations do not fully reproduce the dynamics of a 3D spherical cluster. This is because the electrostatic potential energy contained in a cluster constituted only by ions, and also that per ion, which corresponds to the average kinetic energy obtained from the Coulomb explosion, is different between 2D and 3D cases, as discussed in Refs. [26,40]. However, fundamental ingredients of the interaction between a 3D cluster and high power laser are modeled qualitatively when parameters related to laser and cluster are chosen properly. Meanwhile, we need to compare the dynamics obtained by $2 \mathrm{D}$ simulations with that of $3 \mathrm{D}$ and discuss the difference, which is a future work.
On the other hand, it is noted that the 2D configuration of a cluster coincides with that of a rod, which is uniform in the $z$ direction. The present simulation then corresponds to that analyzing the interaction between rod and laser. Such an experiment is also in our future scope.

The rest of this paper is organized as follows. In Sec. II, we describe numerical modelings for the simulations using EPIC3D together with parameters of a laser and a cluster medium consisting of a solid cluster and ambient gas, and also their configurations. In Sec. III, we present simulation results divided into three subsections: an overview of the interaction (Sec. III A), the resulting ion acceleration (Sec. III B), and the establishment of kinetic equilibrium (Sec. III C). In Sec. IV, we discuss the density dependence concerning the evolution of the structure at the boundary layer (Sec. IV A) and the difference of transition times between three successive phases (Sec. IV B). The theoretical background providing the dependence is discussed in Appendices A and B. Our conclusions are given in Sec. V.

\section{NUMERICAL MODELING}

Simulations are performed using a 2D version of EPIC3D [41]. In a series of simulations, transparent boundary conditions are employed for fields and particles in the $x$ and $y$ directions. The simulation parameters replicate the experimental works [42,43]: A cluster medium consists of a single carbon cluster with a radius $a=125 \mathrm{~nm}$, the electron density of which is $n_{e}^{c l}=3.8 \times 10^{23} \mathrm{~cm}^{-3}\left(=227 n_{c}\right)$, and a surrounding ambient hydrogen gas, the electron density of which is $n_{e}^{a g}=4.0 \times 10^{19} \mathrm{~cm}^{-3}\left(=0.024 n_{c}\right)$, where $n_{c}$ is the critical density of the plasma corresponding to the laser wavelength $\lambda=810 \mathrm{~nm}$. The initial cluster radius $a$ is greater than the collisionless electron skin depth $\delta_{e}=11.6 \mathrm{~nm}$, which is defined by $\delta_{e} \equiv c / \omega_{p}$, where $c$ is the speed of light and $\omega_{p} \equiv \sqrt{4 \pi e^{2} n_{e} / \gamma m_{e}}$ (the Lorentz factor $\gamma$ is defined by $\gamma \equiv$ $\sqrt{1+a_{0}^{2} / 2}$ for the linearly polarized laser pulse) is a relativistic plasma frequency, where $a_{0} \equiv e E / m_{e} \omega c$ is the normalized amplitude of the laser. The cluster is reproduced by particlein-cell particles using 5000 per cell. The simulation box size is $L_{x} \times L_{y}=25.60 \times 25.60 \mu \mathrm{m}$ containing $2560 \times 2560$ cells. The cluster is located at the center of the box at $(x, y)=$ $(0,0)$. The ambient hydrogen gas, surrounded by the vacuum region, occupies the region of $-7.68<(x, y)<+7.68 \mu \mathrm{m}$. Note that the cluster and ambient gas come into contact with each other as the initial condition. A linearly polarized laser electric field in the $x$ direction with a pulse duration $\tau=33 \mathrm{fs}$ (FWHM) is generated from an antenna located at $y=-12.78 \mu \mathrm{m}$ and propagates in the $+y$ direction. The peak intensity of the laser pulse is set to $1.0 \times 10^{19} \mathrm{~W} / \mathrm{cm}^{2}$ $\left(a_{0}=2.19\right)$. The pulse peak passes through the center of the cluster at $t=86.7$ fs. For simplicity of analysis, a fully ionized plasma is employed as the initial condition because the laser field is strong enough to generate fully ionized plasmas, i.e., $\mathrm{H}^{+}$and $\mathrm{C}^{6+}$ [44]. Moreover, the plasma is treated as collisionless since the electron temperature is high enough.

\section{DYNAMICS OF THE BOUNDARY LAYER IN BETWEEN THE CLUSTER AND AMBIENT GAS}

Here, we present the overall dynamics of the boundary layer generated by the interaction between cluster and ambient 
Cluster electrons
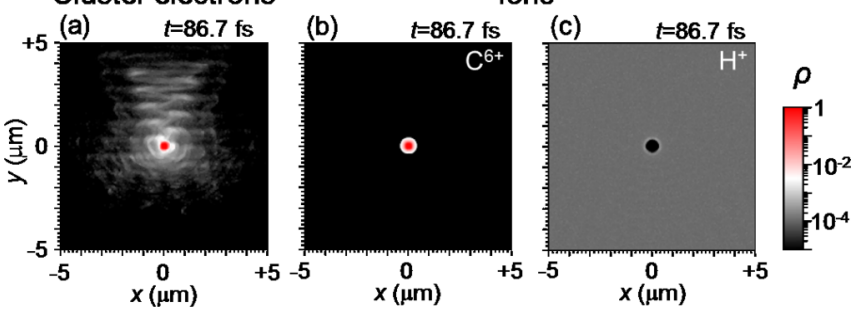

FIG. 1. Snapshots of the 2D images for charge density distributions of (a) the cluster electrons $\rho_{e}^{c l}$, (b) the cluster ions $\rho_{i}^{c l}$, and (c) the ambient gas ions $\rho_{i}^{a g}$ at $t=86.7 \mathrm{fs}$ in the case of the ambient gas density $n_{a g}=0.024 n_{c}$ and the laser amplitude $a_{0}=$ 2.19. Densities are normalized by the initial charge density of the cluster ions and displayed on a log scale.

gas by classifying it into three successive phases in Sec. III A. The dynamics is tightly related to the particle acceleration by nonlinear waves, which we discuss by studying the phase space distribution of ions in Sec. III B. In Sec. IIIC, we discuss the formation of the kinetic equilibrium coupled with the electron dynamics.

\section{A. Overview of the dynamics of the boundary layer}

Figures 1(a)-1(c) show snapshots of 2D images for charge density distributions of the cluster electrons $\rho_{e}^{c l}$ and ions $\rho_{i}^{c l}$ $\left(\mathrm{C}^{6+}\right)$, and the ambient gas ions $\rho_{i}^{a g}\left(\mathrm{H}^{+}\right)$at $t=86.7 \mathrm{fs}$, which is the time at which the peak of the laser pulse reaches the center of the cluster. Here, the charge density distribution of total electrons is given by $\rho_{e}=\rho_{e}^{c l}+\rho_{e}^{a g}$, where $\rho_{e}^{a g}$ represents the charge density distributions of the ambient gas electrons. Note that prior to this time the interaction between the laser pulse and the cluster has taken place approximately from $t=40 \mathrm{fs}$. A significant number of electrons are expelled from the peripheral region of the cluster and move forward ( $+y$ direction) with figure-eight motions by the Lorenz force $\boldsymbol{v} \times \boldsymbol{B}$ as seen in Fig. 1(a). Here, the electron excursion length

\section{Cluster electrons}

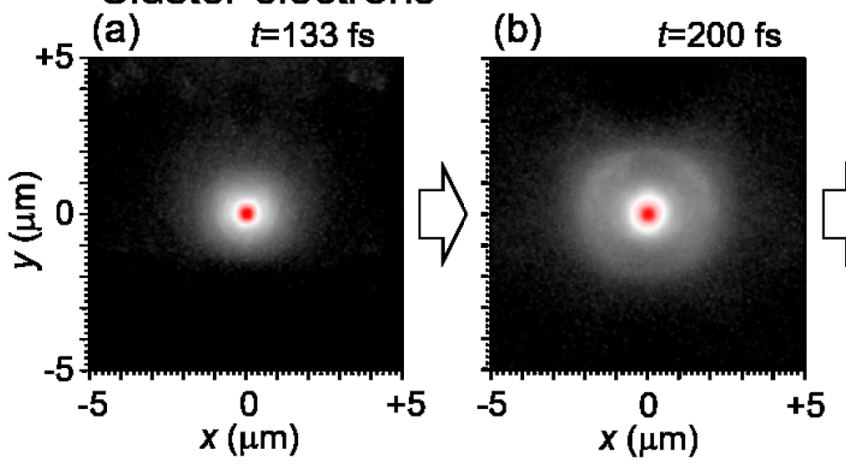

(c)

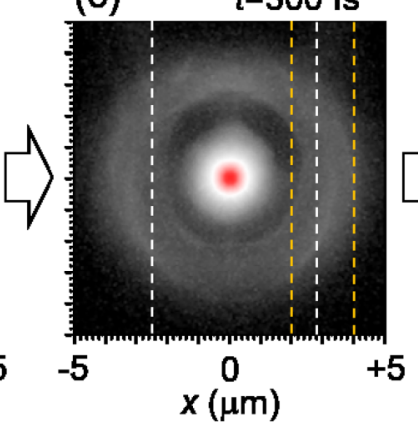

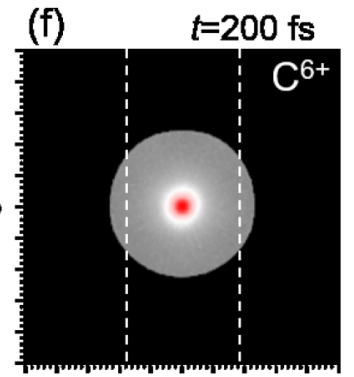

(j)

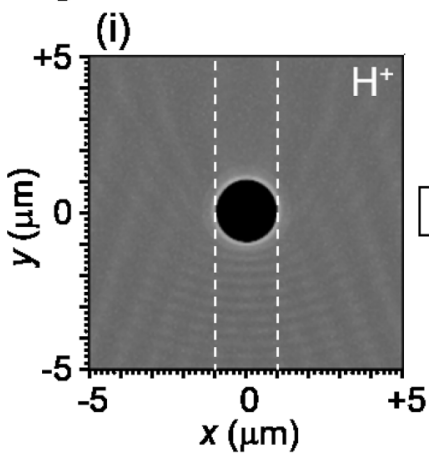
$t=133$ fs
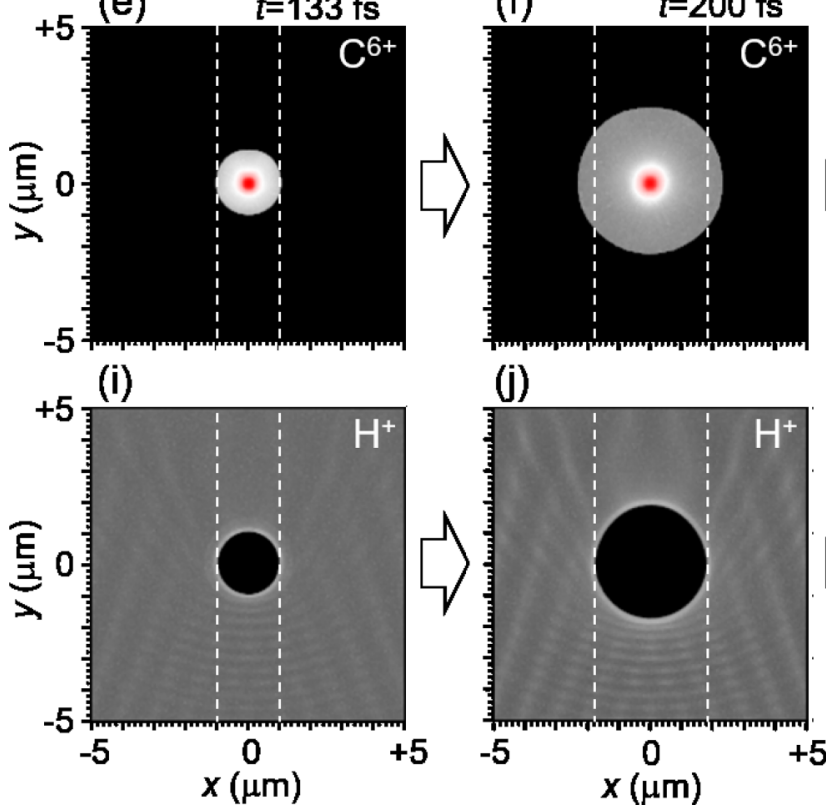

(g)

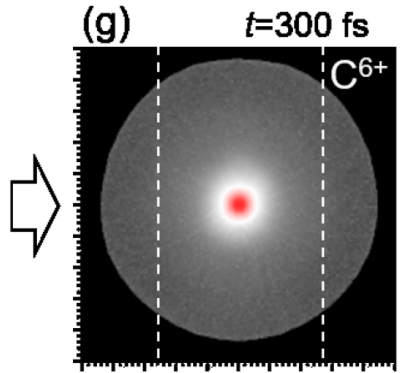

(k)



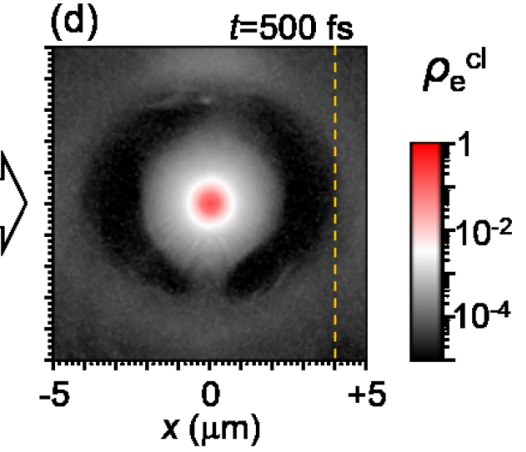

(h)

$t=500$ fs

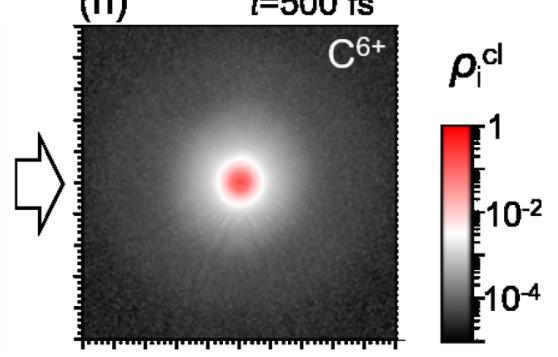

(I)

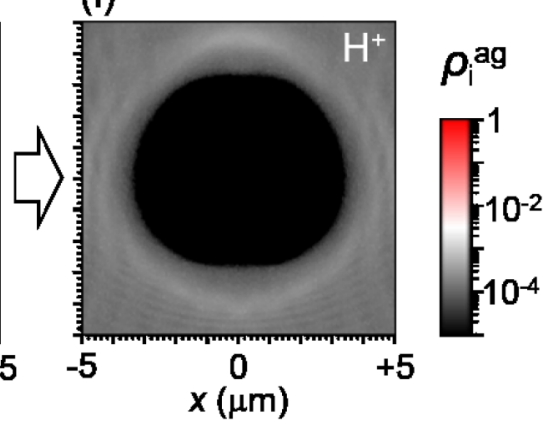

FIG. 2. Snapshots of the 2D images for charge density distributions of (a)-(d) the cluster electrons $\rho_{e}^{c l}$, (e)-(h) the cluster ions $\rho_{i}^{c l}$, and (i)-(1) the ambient gas ions $\rho_{i}^{a g}$ at (a), (e), (i) $t=133 \mathrm{fs}$; (b), (f), (j) $t=200 \mathrm{fs}$; (c), (g), (k) $t=300 \mathrm{fs}$; and (d), (h), (l) $t=500 \mathrm{fs}$ in the case of the density $n_{a g}=0.024 n_{c}$ and the amplitude $a_{0}=2.19$. Densities are normalized by the initial charge density of the cluster ions and displayed on a log scale. White dashed lines show positions of the compressed surface of ambient gas ions in the $x$ direction. Orange dashed lines in (c) and (d) show positions of the ringlike structure of cluster electrons in the $+x$ direction. 
due to the figure-eight motion is approximately given by $\xi_{e} \equiv$ $(c / \omega) \arcsin \left(a_{0} / \sqrt{1+a_{0}^{2}}\right)$, which is about $147 \mathrm{~nm}$ at the peak of the laser pulse $\left(a_{0}=2.19\right)$. Therefore, the relation among $a$, $\xi_{e}$, and $\delta_{e}$ is given by $a \simeq \xi_{e} \gg \delta_{e}$, so that the dynamics roughly belongs to the regime of the ambipolar expansion [26].

Namely, an ambipolar electric field, which is similar to that exhibited around the ion expanding front into vacuum in laser-irradiated plasma causing fast ions [5], is produced around the front of cluster ions and that of ambient gas ions. Consequently, the cluster expands and the ambient gas is compressed in the radial direction as seen in Figs. 1(b) and 1(c). During this process, the front of cluster ions and that of ambient gas ions move outward keeping contact without mixing, which is regarded as a boundary layer in between two distinct media.

Figures 2(a)-2(1) show 2D snapshots for the charge densities, $\rho_{e}^{c l}, \rho_{i}^{c l}$, and $\rho_{i}^{a g}$, at $t=133,200,300$, and $500 \mathrm{fs}$, respectively, as discussed in Fig. 5(a). In order to clearly see the dynamics of the boundary layer, we also show 1D cross sectional views for the charge densities $\rho_{i}^{c l}, \rho_{i}^{a g}, \rho_{e}^{c l}$, and $\rho_{e}^{a g}$, and the resulting electric field $E_{y}$ along the $+y$ direction from the center of the cluster in Figs. 3(a)-3(d). The charge density profiles of ambient gas ions $\rho_{i}^{a g}$ at $t=133,200,250,300$, 340, 400, and 500 fs are also shown in Fig. 3(e). Note that the dynamics of the cluster and the ambient gas are approximately spherically symmetric as seen in Figs. 2(e)-2(1). We find that the dynamics of the boundary layer can be classified into three successive phases as discussed in the following. Here, in order to grasp the positional relation between the cluster expansion front and the compressed surface of the ambient gas, we show schematic views concerning spatial evolutions of the charge densities of cluster ions $\left(\rho_{i}^{c l}\right)$ and ambient gas ions $\left(\rho_{i}^{a g}\right)$, and the phase space distributions of cluster ions and ambient gas ions at representative times in Fig. 4.

Phase 1 ( $t<150 \mathrm{fs}$ ). This is the phase in which the contact surface exists during the expansion of the cluster ions and the compression of the ambient gas ions as shown in Figs. 2(e) and 2(i), which exhibits essentially the same structure as that established in earlier time as discussed in Fig. $1(t=86.7 \mathrm{fs})$. This feature can be seen in Fig. 3(a).

Here, we define the radial location of the cluster expansion front as $r_{f}^{c l}$ with the velocity $v_{f}^{c l}$ and that of the compressed surface of ambient gas as $r_{f}^{a g}$ with the velocity $v_{f}^{a g}$. Then, the contact surface is determined by the relations $r_{f}^{c l} \simeq r_{f}^{a g}$ and $v_{f}^{c l} \simeq v_{f}^{a g}$ [see Fig. 4(c1)]. This phase is the formation process of the shock front, which coincides with the contact surface.

The maximum value of the ambipolar electric field $E_{m}^{a m}$ around the contact surface in the presence of the ambient gas becomes slightly smaller than that in the absence of the ambient gas, i.e., $E_{m 0}^{a m}$, as $E_{m}^{a m} / E_{m 0}^{a m} \sim 0.9$. This is because electrons of the ambient gas flow into the cluster and weaken the charge separation around the cluster expansion front [see blue dotted line in Fig. 3(a)]. Then, the velocity $v_{f}^{c l}$ in the presence of the ambient gas is slightly decreased compared with that in the absence of the ambient gas as discussed in Fig. 5(a). The effect becomes clearer in higher density cases of the ambient gas as discussed in Sec. IV A.
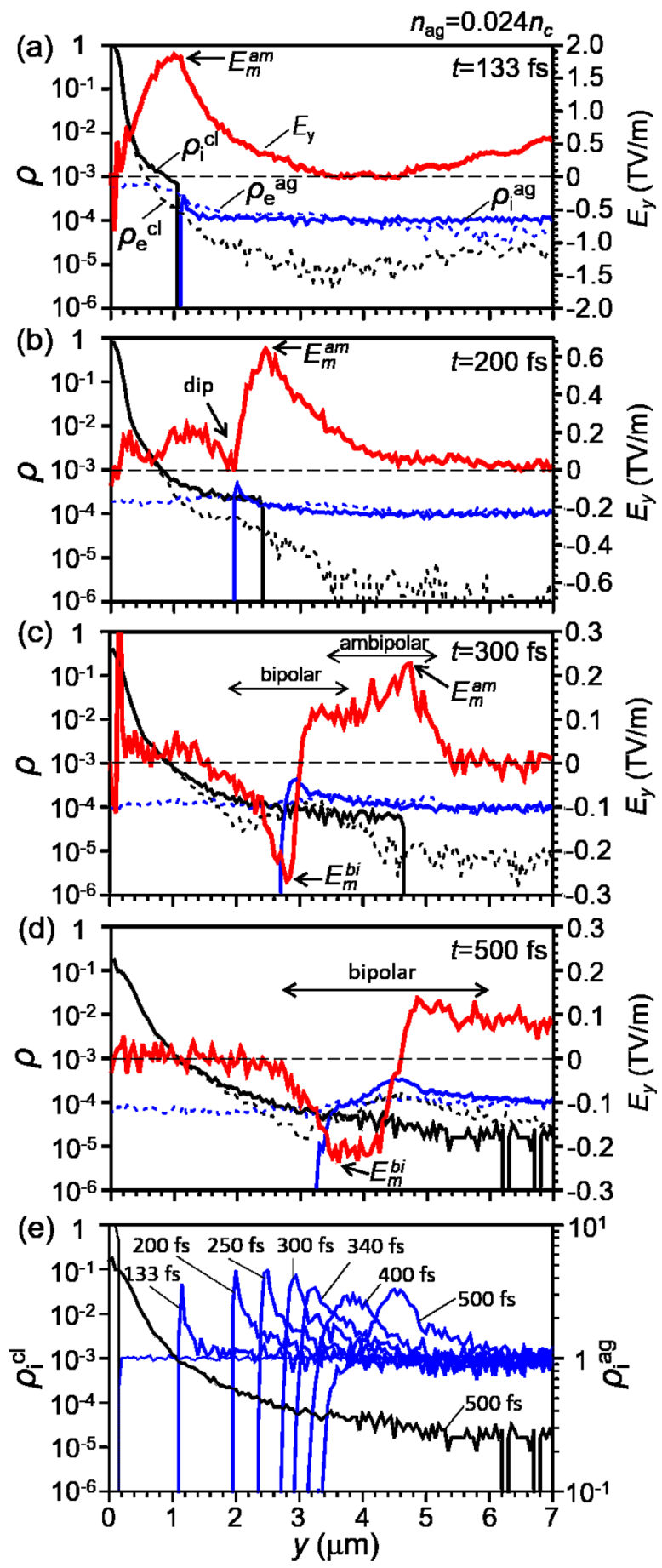

FIG. 3. (a)-(d) The 1D cross sectional views for charge density distributions of cluster ions $\rho_{i}^{c l}$ (black solid line), ambient gas ions $\rho_{i}^{a g}$ (blue solid line), cluster electrons $\rho_{e}^{c l}$ (black dotted line), ambient gas electrons $\rho_{e}^{a g}$ (blue dotted line), and the resulting electric field $E_{y}$ (red solid line) along the $+y$ direction from the center of the cluster at (a) $t=133 \mathrm{fs}$, (b) $t=200 \mathrm{fs}$, (c) $t=300 \mathrm{fs}$, and (d) $t=500 \mathrm{fs}$. The maximum value of the ambipolar electric field $E_{m}^{a m}$ and the maximum negative value of the bipolar electric field $E_{m}^{b i}$ are also shown. Densities are normalized by the initial density of the cluster ions. (e) The time evolution of charge density distributions of cluster ions (black solid line) and ambient gas ions (blue solid line). Dotted lines show the initial charge density distributions of cluster ions (black) and ambient gas ions (blue). 



FIG. 4. (a1)-(f1) Schematic views of the density distribution of cluster ions (black solid lines) and ambient gas ions (blue solid lines). Schematic views of the phase space distributions of cluster ions (green thick lines) and ambient gas ions (red thick lines) corresponding to (a1)-(f1) are shown in (a2)-(f2). A peaking structure is marked by (*). Open and/or filled circles in (b2) and (c2) represent locations of ions.

It is interesting to study the characteristics of the compressed "surface" of the ambient gas. Namely, ions constituting the compressed surface of the ambient gas at a certain time [see ions (P) in Fig. 4(b2)] are accelerated in the forward direction, resulting in escaping from the surface [see also ions (P) in Fig. 4(c2)]. Instead, ions located ahead of the surface [see ions (Q) in Fig. 4(b2)] are accelerated and tend to constitute the new compressed surface [see also ions (Q) in Fig. 4(c2)]. This indicates that the compressed surface is dynamically formed by different ions by exchanging with those located ahead of the surface. On the other hand, the cluster expansion front (surface) is formed by the same ions, whose velocity is the fastest, as seen in Figs. 4(b2) and 4(c2).

Phase 2 (150 fs $\leqslant t<250 \mathrm{fs}$ ). This is the phase in which the crossing between the cluster expansion front and the compressed surface of the ambient gas takes place, leading to an overlapping region, i.e., $v_{f}^{a g}<v_{f}^{c l}$ and $r_{f}^{a g}<r_{f}^{c l}$ [see Fig. 4(d1)]. This region becomes wider with time as seen in panel (f) of Fig. 2 and panels (g), (j), and (k). In the following, we investigate the process of the crossing in detail.

In the early time of the interaction between the laser pulse and the cluster, the charge density of cluster ions at the expansion front $\rho_{i, f}^{c l}$ is much higher than that of the ambient gas ions at the compressed surface $\rho_{i, f}^{a g}$, i.e., $\rho_{i, f}^{c l} / \rho_{i, f}^{a g} \sim 10^{4}$ [see Fig. 4(b1)]. However, after the main part of the laser pulse hits the cluster, the ratio quickly decreases with time, e.g., down to $\rho_{i, f}^{c l} / \rho_{i, f}^{a g} \sim 5$ at $t=133$ fs as seen in Fig. 3(a). On the other hand, the electron charge density at around the contact surface $\rho_{e}$, which is almost spatially uniform since the electron temperature is high enough $(\sim 0.4 \mathrm{MeV})$, is higher than the charge density $\rho_{i, f}^{a g}$ while lower than the charge density $\rho_{i, f}^{c l}$, such that $\rho_{i, f}^{a g}<\rho_{e}<\rho_{i, f}^{c l}$ as also seen in Fig. 3(a). Then, the discontinuity between the charge density $\rho_{i, f}^{c l}$ and the charge density $\rho_{i, f}^{a g}$ produces the ambipolar electric field $E_{y}$ around the contact surface as discussed in phase 1. Here, it is noted that a small gap exists in between $r_{f}^{c l}$ and $r_{f}^{a g}$ (at $y=1.1 \mu \mathrm{m})$ as seen in Fig. 3(a) $(t=133 \mathrm{fs})$, so that $r_{f}^{c l}<r_{f}^{a g}$ [see Fig. 4(a1)]. This is because the ambient gas ions $\mathrm{H}^{+}$are more accelerated by the field $E_{y}$ than the cluster ions $\mathrm{C}^{6+}$ due to the difference of the charge to mass ratio between $\mathrm{H}^{+}$ and $\mathrm{C}^{6+}$.

As time goes on, the maximum value of the field $E_{m}^{a m}$ is gradually reduced since the charge density $\rho_{i, f}^{c l}$ also decreases due to the cluster expansion while the charge density $\rho_{i, f}^{a g}$ increases due to the compression of the ambient gas. Note 

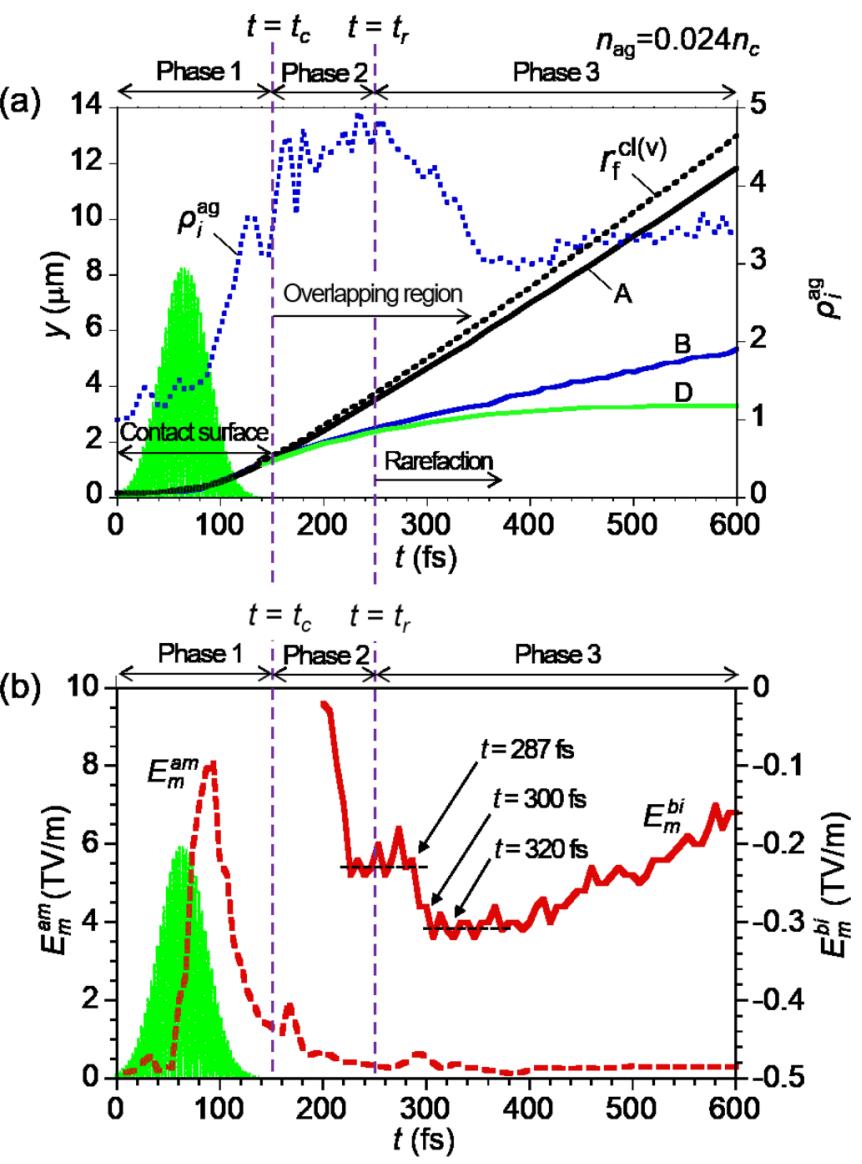

FIG. 5. (a) Temporal evolutions for the location of the expansion front of the cluster ions $r_{f}^{c l}$ (black solid line, A), the location exhibiting the peak density of ambient gas ions $r_{f(B)}^{a g}$ (blue solid line, B), and that exhibiting the front of ambient gas ions $r_{f(D)}^{a g}$ (green solid line, D). Here, A, B, and D represent the same fronts as those in Fig. 12. The charge density of the peaking structure of the ambient gas ions (blue dotted line) along the $+y$ direction from the center of the cluster is also shown. The black dashed line represents the temporal evolution for the location of the expansion front of the cluster ions in a vacuum $r_{f}^{c l(v)}$ as a reference. The green dotted line represents the intensity of the laser pulse. The pulse peak passes through the center of the cluster at $t=86.7 \mathrm{fs}$. The charge density of the peaking structure of the ambient gas ions is normalized by the initial charge density of the ambient gas ions. (b) Temporal evolutions for the maximum value of the ambipolar electric field $E_{m}^{a m}$ (red dotted line) and the maximum negative value of the bipolar electric field $E_{m}^{b i}$ (red solid line). The intensity of the laser pulse is also shown (green dotted line).

that the compression leads to a peaking structure with a sharp edge at the front of the ambient gas as seen in Fig. 3(a) and/or as schematically illustrated in Figs. 4(b1) and 4(c1) [marked by $(*)$ ]. Consequently, the discontinuity between the charge densities $\rho_{i, f}^{c l}$ and $\rho_{i, f}^{a g}$, i.e., $\rho_{i, f}^{c l} / \rho_{i, f}^{a g}$, gradually becomes small from $10^{4}$ down to 1 at $t \sim 150$ fs [see Figs. 4(a1), 4(b1), and 4(c1)]. Accordingly, the field $E_{y}$ decreases while the cluster expansion front is further accelerated, so that incoming ambient gas ions constituting the ambient gas surface overtake the electrostatic potential produced by the field $E_{y}$, leading to the crossing between the cluster expansion front and the ambient gas surface [see Fig. 3(b)]. This mechanism is discussed in Sec. III B. Namely, the crossing is triggered when the charge density of the cluster ions and that of the ambient gas ions roughly coincide at the contact surface as $\rho_{i, f}^{a g} \simeq \rho_{i, f}^{c l}$. We find that this condition is fulfilled in a wide range of ambient gas density, which we discuss in Sec. IV B.

After the crossing, the charge density of the sharp peaking structure $\rho_{i, f}^{a g}$ still keeps increasing and reaches the maximum value at $t \sim 250 \mathrm{fs}$ as seen in Fig. 3(e) and also in Fig. 5(a). It is noted that during this process, the field $E_{y}$ changes the structure qualitatively. Namely, a dip structure in the field $E_{y}$ emerges at the left-hand side of the compressed surface of the ambient gas $(y \sim 2.0 \mu \mathrm{m})$ as seen in Fig. 3(b), which results from the net positive charge around the compressed surface. As a result, in addition to the ambipolar structure around the cluster expansion front, a bipolar structure having the maximum negative value $E_{m}^{b i}$ around the compressed surface is established as seen in Fig. 3(c). Note that these two structures are connected in the overlapping region.

Phase $3(250 \mathrm{fs} \leqslant t)$. This is the phase in which the sharp peaking structure of the compressed surface tends to be relaxed [see Figs. 2(1) and 4(e1)].

The density profile of ambient gas ions in Figs. 3(c) and 3 (d) is found to be qualitatively different from those seen in Figs. 3(a) and 3(b) exhibiting the sharp peaking structure. This indicates that a different dynamics is triggered in the ambient gas in the time between Figs. 3(b) and 3(c). In order to see this process clearly, we study density profiles of ambient gas ions in Fig. 3(e). It can be seen that the sharp peaking structure of the compressed surface, which is maintained up to $t \sim 250 \mathrm{fs}$, starts to collapse after $t \sim 250$ fs [see also blue dotted line in Fig. 5(a)], and then relaxed with time, leading to the formation of the region with a gentle gradient [see Fig. 3(d)]. In order to characterize the structure of the ambient gas, we define the location exhibiting the peak density $r_{f(B)}^{a g}$ and that of the front of the ambient gas $r_{f(D)}^{a g}$. Namely, the sharp peaking structure of the ambient gas ions is collapsed and bifurcated into two components; i.e., one is $r_{f(B)}^{a g}$ and the other is $r_{f(D)}^{a g}$. This feature is illustrated in Figs. 4(e1) and 4(f1).

After $t \sim 250 \mathrm{fs}, r_{f(B)}^{a g}$ is found to propagate in the $+y$ direction with a velocity $v_{f(B)}^{a g}$ roughly the same as the ion sound velocity $\left(\sim 1.35 c_{s} ; c_{s}\right.$ : ion sound velocity). On the other hand, $r_{f(D)}^{a g}$ also propagates in the $+y$ direction while with the velocity $v_{f(D)}^{a g}$ slower than $c_{s}$. Therefore, the region with a gentle gradient, whose width is given by $r_{f(B)}^{a g}-r_{f(D)}^{a g}$, becomes wider with time as seen in Fig. 5(a). From these observations, the dynamics of the relaxation of the sharp peaking profile of the ambient gas is considered to result from the onset of a rarefaction wave and propagation. This dynamics is clearly observed when the density of the ambient gas is increased, as we discuss in Sec. IV A (see Fig. 11). Note that the onset of the rarefaction wave is found to coincide with the situation in which some of the ambient gas ions start to pass over the electrostatic potential at the compressed surface of the ambient gas ions, which we discuss in Sec. III B. 

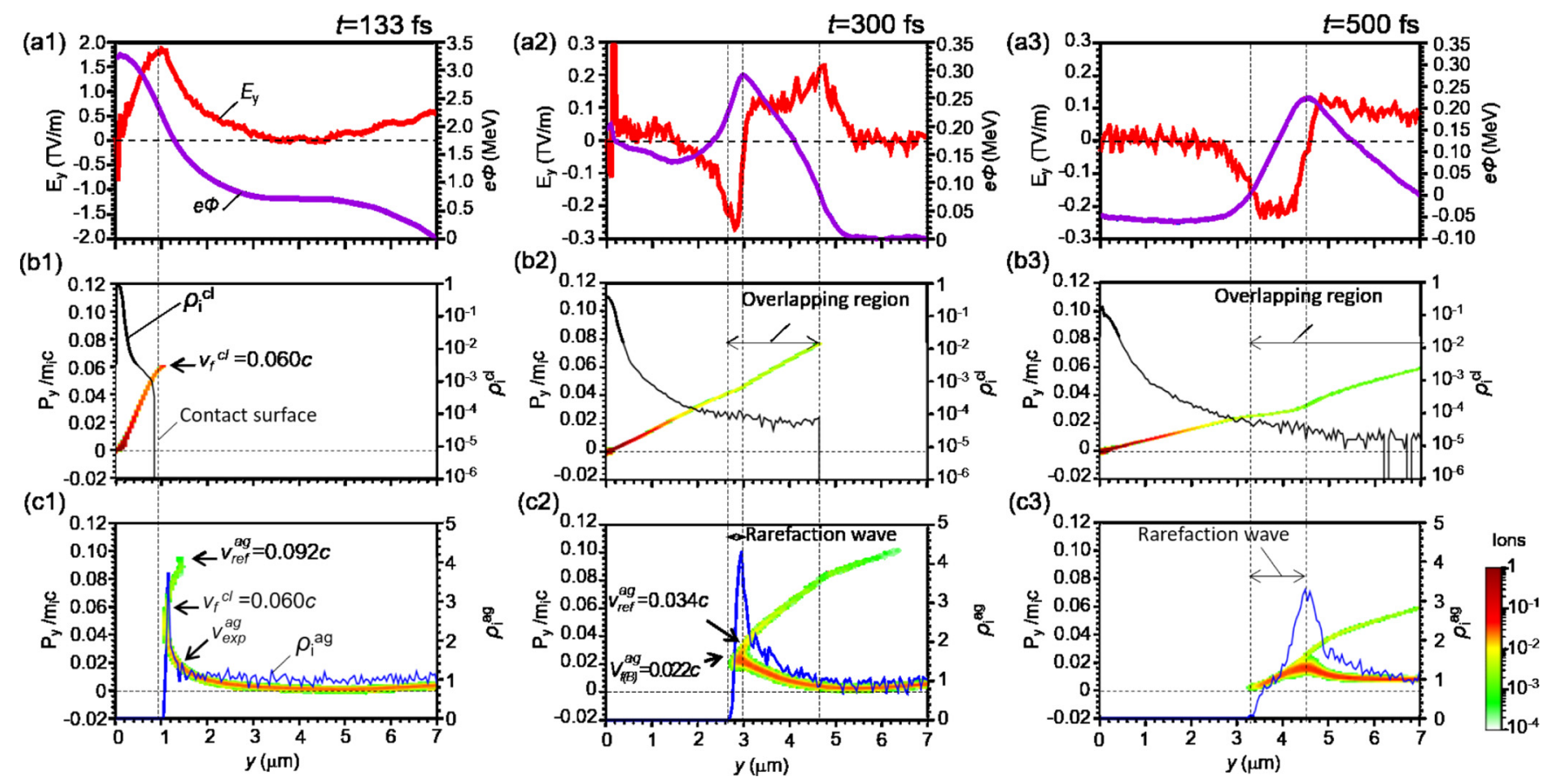

FIG. 6. The 1D cross sectional views for (a1)-(a3) the field $E_{y}$ (red solid line) and the electrostatic potential for ions $e \Phi$ (purple solid line), (b1)-(b3) the charge density $\rho_{i}^{c l}$ (black solid line) and the phase space distributions of the cluster ions, (c1)-(c3) the charge density $\rho_{i}^{a g}$ (blue solid line) along the $+y$ direction from the center of the cluster at (a1)-(c1) $t=133 \mathrm{fs},(\mathrm{a} 2)-(\mathrm{c} 2) t=300 \mathrm{fs}$, and (a3) $-(\mathrm{c} 3) t=500 \mathrm{fs}$. Electrostatic potentials are set to zero at $y=7 \mu \mathrm{m}$. Charge densities are normalized by the initial density of ions. Phase space distributions consist of ions in the region of $y \geqslant 0$ and $-0.15 \leqslant x \leqslant+0.15 \mu \mathrm{m}$, and are normalized by the initial charge density of the cluster ions and displayed on a log scale.

It is interesting to study the density distributions of cluster electrons in Fig. 2. Namely, a ringlike structure is found to be formed for the density distribution of cluster electrons in $2 \leqslant$ $r \leqslant 4 \mu \mathrm{m}$ at $t=300 \mathrm{fs}$ as seen in Fig. 2(c), indicating that cluster electrons are trapped. The structure is considered to connect with the establishment of kinetic equilibrium, which we discuss in detail in Sec. III C.

Transition dynamics for the boundary layer. Here, we summarize the above dynamics classified into three successive phases in Fig. 5. Figure 5(a) illustrates temporal evolutions for the location of the expansion front of the cluster ions $r_{f}^{c l}$, that exhibiting the peak density of ambient gas ions $r_{f(B)}^{a g}$, and that exhibiting the front of ambient gas ions $r_{f(D)}^{a g}$. The charge density of the peaking structure of the ambient gas ions $\rho_{i}^{a g}$ is also shown. The green dotted line represents the intensity of the laser pulse. The location of the cluster expansion front in a vacuum $r_{f}^{c l(v)}$, i.e., the case of a single cluster expansion in the absence of the ambient gas, is also shown as a reference. Figure 5(b) shows temporal evolutions for the maximum value of the ambipolar electric field $E_{m}^{a m}$ and the maximum negative value of the bipolar electric field $E_{m}^{b i}$. The details are discussed in Sec. III C. Here, we define the time at which the crossing between the cluster expansion front and the compressed surface of the ambient gas takes place as $t=t_{c}$, and the time at which the rarefaction wave is triggered as $t=t_{r}$. In the present case, these times are approximately $t_{c} \sim 150 \mathrm{fs}$ and $t_{r} \sim 250 \mathrm{fs}$, respectively. Hereafter, we refer to the times $t_{c}$ and $t_{r}$ as the "crossing time" and "trigger time," respectively. In phase 1, the cluster expansion front (velocity $v_{f}^{c l}$ ) and the compressed surface of the ambient gas (velocity $v_{f}^{a g}$ ) move outward together for $0 \leqslant t<t_{c}$ as seen in Fig. 5. In phase 2, the cluster expansion front moves ahead of the compressed surface of the ambient gas [see also Fig. 4(d1)]. In phase 3 , the compressed surface of the ambient gas starts to evolve as a rarefaction wave. In this phase, the width represented by $r_{f(B)}^{a g}-r_{f(D)}^{a g}$ in Fig. 5 is found to become wider with time as the result of the propagation of the rarefaction wave [see Figs. 4(e1) and 4(f1)].

\section{B. Ion acceleration by nonlinear waves}

Here, it is noted that the dynamics discussed in Sec. III A is tightly related to that of the acceleration for ambient gas ions. This is because an electrostatic potential formed by the amibipolar electric field produced around the contact surface seen in Figs. 3(a)-3(c) moves outward and reflects ambient gas ions (as discussed in phase 2 in Sec. III A). To understand this feature, we study the dynamics of both cluster ions and those of ambient gas in more detail by investigating their phase space distributions. In Fig. 6, we show 1D cross sectional views for the field $E_{y}$ and the potential energy for ions $e \Phi$ [panels (a1)-(a3)] and the charge density $\rho_{i}$ and the phase space distributions of cluster ions [panels (b1)-(b3)] and ambient gas ions [(c1)-(c3)] at three different times, i.e., $t=133,300$, and $500 \mathrm{fs}$. We find that the electric field patterns that evolve from ambipolar structure to bipolar structure in the three successive phases contribute to the ion acceleration in two different ways, i.e., phase 1: acceleration 
by a collisionless shock, and phases 2 and 3: acceleration by a nonlinear wave, which we discuss in the following.

Acceleration by collisionless shock. In phase $1\left(t<t_{c}\right)$, i.e., before the crossing time $t_{c}$, the ambient gas ions are compressed and reflected by the electrostatic potential associated with the ambipolar electric field at the contact surface. Namely, a collisionless shock acceleration of ambient gas ions takes place as seen in Figs. 6(a1)-6(c1), which is the case at $t=133 \mathrm{fs}$.

In this case, the propagation velocity of the shock front, which corresponds to the velocity of the cluster expansion front, is $v_{f}^{c l} \simeq 0.060 c\left(1.8 \times 10^{7} \mathrm{~m} / \mathrm{s}\right)$, while the ion sound velocity, defined by $c_{s} \equiv \sqrt{2 k_{B}\left(T_{e}+T_{i}\right) / m_{i}}$, is estimated as $c_{s} \simeq 0.029 c\left(8.7 \times 10^{6} \mathrm{~m} / \mathrm{s}\right)$ at the upstream region, i.e., $y=$ $7 \mu \mathrm{m}$, where $k_{B}$ is the Boltzmann constant, $T_{e}$ and $T_{i}$ are the temperatures of electrons and cluster ions, respectively, and $m_{i}$ is the proton mass. The estimated shock Mach number $\mathcal{M}$ is approximately $\mathcal{M}=2.1$, which exceeds the critical value for the collisionless shock acceleration, i.e., $\mathcal{M}>1.6$ [45].

In collisionless shock acceleration in plasmas, the shock front accelerates upstream ions, i.e., the ambient gas ions in the present case, leading to an ion beam with a narrow energy spread $[9,11]$, by reflecting them to double the shock velocity. In the condition in which upstream ions are pre-accelerated by the front part of the electric field associated with the shock, upstream ions have the velocity $v_{u p}$ prior to the shock acceleration. They are reflected by the shock potential when the kinetic energy of upstream ions $K$, which is represented by

$$
K=\frac{1}{2} m_{i}\left(v_{u p}-v_{s h}\right)^{2},
$$

is less than the potential energy of the shock $e \Phi$, i.e., $K<e \Phi$, in the shock rest frame. Here, $v_{s h}$ represents the shock velocity. As a result, reflected ions are accelerated with the velocity $v_{\text {ref }}=2 v_{s h}-v_{u p}$.

Applying this scenario in the present case, the velocities $v_{s h}, v_{u p}$, and $v_{\text {ref }}$ in Eq. (1) correspond to $v_{f}^{c l}, v_{\text {exp }}^{a g}$, and $v_{\text {ref }}^{a g}$ in Eq. (2), respectively, so that the corresponding kinetic energy of upstream ambient gas ions $K_{1}$ (in the shock rest frame) is represented by

$$
K_{1}=\frac{1}{2} m_{i}\left(v_{\mathrm{exp}}^{a g}-v_{f}^{c l}\right)^{2} .
$$

Here, the velocity $v_{\text {exp }}^{a g}$ represents that of the upstream ambient gas ions, which are pre-accelerated by the front part of the ambipolar field [12] prior to the shock acceleration as seen in the region $1 \leqslant y \leqslant 3 \mu \mathrm{m}$. The velocity defined by $v_{\text {ref }}^{a g}=$ $2 v_{f}^{c l}-v_{\exp }^{a g}$ represents that of reflected ambient gas ions. In the present case, the velocities $v_{f}^{c l}$ and $v_{\text {ref }}^{a g}$ are found to be estimated as $v_{f}^{c l} \simeq 0.060 c$ and $v_{\text {ref }}^{a g} \simeq 0.092 c$, respectively, from the results in Figs. 6(b1) and 6(c1). Then, the velocity $v_{\text {exp }}^{a g}$ is calculated as $v_{\exp }^{a g} \simeq 0.028 c$. One can find this velocity in the phase space distribution of ambient gas ions around the location indicated by an arrow in Fig. 6(c1). Moreover, the kinetic energy of upstream ambient gas ions $K_{1}$ is estimated as $K_{1} \simeq 0.51 \mathrm{MeV}$ by using Eq. (2). This value is smaller than the potential energy $e \Phi \simeq 0.78 \mathrm{MeV}$ [see Fig. 6(a1)], which is also consistent with the condition of the shock acceleration, i.e., $K_{1}<e \Phi$.
The shock acceleration is found to continue up to the time that the crossing between the cluster expansion front and the compressed surface of the ambient gas takes place, i.e., the crossing time $t_{c}$ [see Fig. 4(c1)]. This is because the kinetic energy $K_{1}$ is always less than the potential energy $e \Phi$, i.e., $K_{1}<e \Phi$, up to the crossing time $t_{c}$, though the peak value of ambipolar field, which provides the potential height associated with the shock, is reduced with time [see Figs. 6(a1) and 6(a2)]. However, over this time, i.e., $t>t_{c}$, the condition between the potential energy $e \Phi$ and the kinetic energy $K_{1}$ at the cluster expansion front $y=r_{f}^{c l}$ changes from $K_{1}<e \Phi$ to $K_{1}>e \Phi$, so that the incoming ambient gas ions are no longer reflected at the cluster expansion front, while they continue to climb up the potential across the front surface toward inside the cluster as illustrated in Figs. 4(d1) and 4(d2). The ambient gas ions are then reflected at the compressed surface and accelerated in a manner similar to the reflection at the contact surface in phase 1 . Namely, though the reflection and acceleration of the ambient gas ions by the shock cease due to the disappearance of the shock front by the crossing, the compressed surface of the ambient gas ions plays a role similar to that of the shock front after the crossing time $t_{c}$.

Acceleration by nonlinear wave. In phases 2 and $3\left(t_{c} \leqslant t\right)$, ambient gas ions are reflected by the electrostatic potential associated with the bipolar field around the compressed surface as seen in Figs. 6(a2)-6(c2). Namely, the compressed surface is found to propagate with the velocity $v_{f}^{a g}$ before the trigger time $t_{r}$ [see Fig. 4(d1)]. However, after this time, i.e., $t \geqslant t_{r}$, the compressed surface is found to propagate with the velocity $v_{f(B)}^{a g}$ [see Figs. $4(\mathrm{e} 1)$ and $\left.4(\mathrm{f} 1)\right]$ in the $+y$ direction as a nonlinear wave and causes a similar ion acceleration to that by the shock as seen in Figs. 6(a2)-6(c2), which is the case at $t=300 \mathrm{fs}$ (phase 3).

In the present case, the velocities $v_{s h}, v_{u p}$, and $v_{\text {ref }}$ in Eq. (1) correspond to $v_{f(B)}^{a g}, v_{\mathrm{exp}}^{a g}$, and $v_{\text {ref }}^{a g}$ in Eq. (3), respectively, so that the corresponding kinetic energy of upstream ambient gas ions $K_{2}$ (in the shock rest frame) is represented by

$$
K_{2}=\frac{1}{2} m_{i}\left(v_{\mathrm{exp}}^{a g}-v_{f(B)}^{a g}\right)^{2},
$$

where the velocity $v_{\exp }^{a g}$ has the same definition as that in Eq. (2). Here, some of the ambient gas ions are reflected by the potential that moves with the velocity $v_{f(B)}^{a g} \simeq 0.022 c$ and are accelerated to the velocity $v_{\text {ref }}^{a g} \simeq 0.034 c$, while the rest pass over the potential around the compressed surface at $y=3 \mu \mathrm{m}$ as seen in Figs. 6(a2) and 6(c2). Then, the velocity $v_{\exp }^{a g}$ is calculated as $v_{\text {exp }}^{a g} \simeq 0.010 c$ from the relation $v_{\text {ref }}^{a g}=2 v_{f(B)}^{a g}-$ $v_{\exp }^{a g}$. One can find this velocity in the phase space distribution of ambient gas ions around the location indicated by an arrow in Fig. 6(c2). Here, by using the velocities $v_{f(B)}^{a g} \simeq 0.022 c$ and the ion sound velocity $c_{s} \simeq 0.020 c$, the estimated shock Mach number $\mathcal{M} \equiv v_{f(B)}^{a g} / c_{s}$ is approximately $\mathcal{M}=1.1$, which is below the critical value for the collisionless shock acceleration [45].

As time goes on, almost all ambient gas ions pass over the potential created at the location exhibiting the peak density of the ambient gas ions at $y=4.5 \mu \mathrm{m}$ as seen in Figs. 6(a3)6(c3), which is the case at $t=500 \mathrm{fs}$. This is because the potential height continuously decreases with time, so that 



FIG. 7. Energy spectra of the cluster ions (black solid line) and the reflected ambient gas ions (blue solid line) at (a) $t=133 \mathrm{fs}$ and (b) $t=300 \mathrm{fs}$. The ion number is evaluated in real value by multiplying the particle weight by the PIC particle. Spectra consist of ions in the region of $y \geqslant 0$ and $-0.15 \leqslant x \leqslant+0.15 \mu \mathrm{m}$.

the kinetic energy of upstream ions $K_{2}$ becomes larger than the potential energy $e \Phi$, i.e., $K_{2}>e \Phi$, after $t=t_{r} \sim 250 \mathrm{fs}$, which is the time that the rarefaction wave is triggered as discussed in Sec. III A (phase 3). Namely, some of the incoming ambient gas ions change the characteristics of their orbits from reflection to passing at $t=t_{r}$, leading to the structure of the rarefaction wave.

Energy spectra of ions. Figure 7 shows the energy spectra of cluster ions and reflected ambient gas ions at $t=133$ and $300 \mathrm{fs}$, respectively. Here, we define the reflected component of ambient gas ions in energy spectra as ions having larger velocities than the shock velocity $v_{f}^{c l}$ and/or that of the nonlinear wave $v_{f}^{a g}$. That is, in Fig. 7(a), the spectrum of ambient gas ions consists of those having velocities over $v_{f}^{c l} \simeq 0.060 c$ [see Fig. 6(b1)]. On the other hand, in Fig. 7(b), the spectrum of ambient gas ions consists of those having velocities over $v_{f(B)}^{a g} \simeq 0.022 c$ [see Fig. 6(c2)].

At $t=133 \mathrm{fs}$ [Fig. 7(a)], the shock acceleration is dominant. Ideally, the shock front, i.e., the cluster expansion front in the present case, accelerates upstream ions by reflecting them to double the shock velocity. Then, the ratio of the maximum kinetic energy per nucleon of reflected ambient gas ions $W_{m}^{a g}$ to that of the cluster ions $W_{m}^{c l}$, i.e., $W_{m}^{a g} / W_{m}^{c l}$, becomes 4.0. In the present case, as discussed in Figs. 6(b1) and $6(\mathrm{c} 1)$, the maximum velocity of reflected ambient gas ions $v_{\text {ref }}^{a g}(\simeq 0.092 c)$ is less than twice that of the cluster expansion front $2 v_{f}^{c l}(\simeq 2 \times 0.060 c=0.12 c)$. Therefore, as seen in Fig. 7(a), the maximum kinetic energies $W_{m}^{a g}$ and $W_{m}^{c l}$ are $4.0 \mathrm{MeV} / \mathrm{u}$ and $1.8 \mathrm{MeV} / \mathrm{u}$, respectively, and $W_{m}^{a g} / W_{m}^{c l}$ becomes approximately 2.3 .

At $t=300 \mathrm{fs}$ [Fig. 7(b)], the nonlinear wave acceleration is dominant. In this time, the spectrum of reflected ions shows a wide energy spread ranging from 0.2 to $4.7 \mathrm{MeV} / \mathrm{u}$ with having a small hump at the higher energy side around 3.4 $\mathrm{MeV} / \mathrm{u}$. Here, the energy spread of $0.2 \leqslant E \leqslant 3 \mathrm{MeV} / \mathrm{u}$ results from the further acceleration by the electrostatic field around the overlapping region $(3 \leqslant y \leqslant 4.6 \mu \mathrm{m})$ in Fig. $6(\mathrm{c} 2)$ after the reflection by the potential associated with the nonlinear wave at around $y=3 \mu \mathrm{m}$ in Fig. 6(c2). The hump at around $3.4 \mathrm{MeV} / \mathrm{u}$ originates from the early-phase shock acceleration, i.e., the component ranging from 1.8 to $4.0 \mathrm{MeV} / \mathrm{u}$ in Fig. 7(a). This spectrum shape is consistent with that observed in the experiment [43].

\section{Formation of quasistationary kinetic equilibrium}

In Secs. III A and III B, we found that a boundary layer in between cluster and ambient gas evolves while changing the characteristics qualitatively through transitions from the structure with contact surface (phase 1) through that with overlap (phase 2) to that with a rarefaction wave (phase 3 ). Here, it is noted that these dynamics are dominated by the slow timescale ion motion in phase space in two plasmas coming into contact with each other. On the other hand, fast timescale electron dynamics plays an essential role in determining the electric field that drives the above ion dynamics. In the following, we study such electron dynamics.

Phase space dynamics of electrons. In Fig. 8, we show the momentum distribution of electrons in the phase space together with the electrostatic potential energy $-e \Phi$ at $t=$ 133, 200, and $300 \mathrm{fs}$ in panels (a1), (a2), and (a3), respectively, which is a key leading to the bipolar electric field structure discussed in Sec. III A. Corresponding electron orbits in the phase space with different energies are also shown in Figs. 8(b1), 8(b2), and 8(b3), respectively. Here, the electron trajectories are calculated from $H_{e} \equiv(\gamma-1) m_{e} c^{2}-e \Phi$, where $H_{e}$ is the total energy of an electron. In earlier times as seen at $t=133$ (phase 1) and $200 \mathrm{fs}$ (phase 2), the potential energy $-e \Phi$ (gray solid line) in Figs. 8(a1) and 8(a2) monotonically increases from $y=0$, which confines low energy electrons around the cluster center as seen in Figs. 8(b1) and 8(b2).

On the other hand, as time goes on, the structure of the potential $-e \Phi$ qualitatively changes from the monotonic structure [Fig. 8(a2)] at $t=200 \mathrm{fs}$ (phase 2) to that with two potential wells, i.e., a relatively shallow one at around $y=0$ and a deep one at around $y=2.9 \mu \mathrm{m}$, separated by a hill at around $y=1.6 \mu \mathrm{m}$ [Fig. 8(a3)] at $t=300 \mathrm{fs}$ (phase 3 ). Namely, during the evolution from $t=200 \mathrm{fs}$ (phase 2) [Fig. 8(a2)] to $t=300 \mathrm{fs}$ (phase 3) [Fig. 8(a3)], a separatrix whose $\mathrm{X}$ point is located at around $y=1.6 \mu \mathrm{m}$ is formed as seen in Fig. 8(a3), which leads to the topological change of the electron trajectories. Note that these two potential wells are then embedded in a global potential well ranging from $y=0$ to $y=5 \mu \mathrm{m}$.

Evolution of electric fields. In the above, we investigate the potential structure at three different times corresponding to phases 1,2 , and 3 . Here, we study the temporal evolution of the electric fields, i.e., the ambipolar field having the maximum value $E_{m}^{a m}$ at around the cluster expansion front and the bipolar one having the maximum negative value $E_{m}^{b i}$ at around the compressed surface of the ambient gas, focusing on the timescale.

The value $E_{m}^{a m}$ rapidly decreases until the crossing time $t_{c}$ (phase 1) during the time in which the shock wave survives as discussed in Sec. III B, while it slowly decreases after the crossing time $t_{c}$ (phases 2 and 3). On the other hand, the value $E_{m}^{b i}$ starts to develop after $t \sim 200 \mathrm{fs}$ (phases 2 and 3 ). As the overlapping region between the cluster expansion front and the compressed surface of the ambient gas becomes wider [see Fig. 5(a)], the value $E_{m}^{b i}$ reaches to 

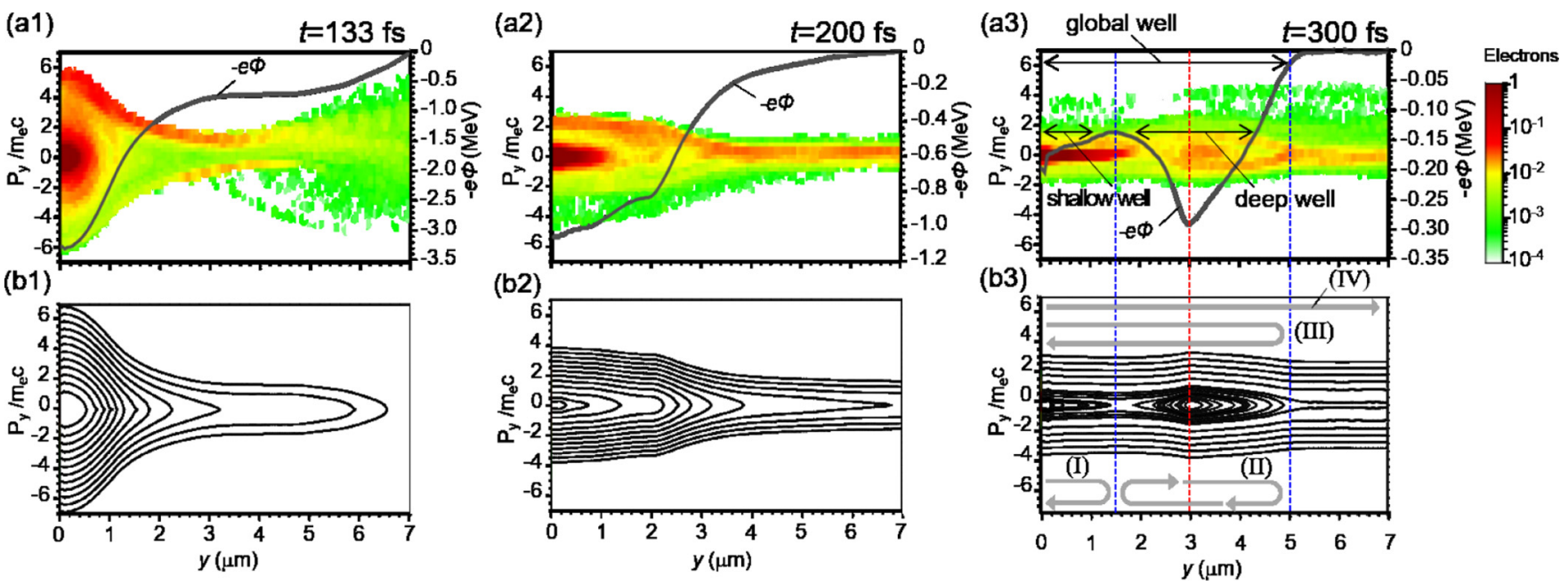

FIG. 8. The 1D cross sectional views for (a1)-(a3) the phase space distributions of electrons and the electrostatic potential for electrons $-e \Phi$ (gray solid line), and (b1)-(b3) the contour plots of electron trajectories along the $+y$ direction from the center of the cluster at (a1), (b1) $t=133 \mathrm{fs}$, (a2), (b2) $t=200 \mathrm{fs}$, and (a3), (b3) $t=300 \mathrm{fs}$. The electrostatic potential is set to zero at $y=7 \mu \mathrm{m}$. Roman numerals (I)-(IV) in (b3) represent typical electron trajectories corresponding to the potential $-e \Phi$ in (a3). Phase space distributions consist of electrons in the region of $y \geqslant 0$ and $-0.15 \leqslant x \leqslant+0.15 \mu \mathrm{m}$, and are normalized by the initial charge density of the cluster electrons and displayed on a log scale.

around $E_{m}^{b i} \simeq-0.23 \mathrm{TV} / \mathrm{m}$ at $t \sim 220$ fs. Interestingly, at $t \sim 290 \mathrm{fs}$, the value $E_{m}^{b i}$ again develops to another constant value around $E_{m}^{b i} \simeq-0.31 \mathrm{TV} / \mathrm{m}$. After $t \sim 400 \mathrm{fs}$, the value $E_{m}^{b i}$ is gradually decreased. In addition, during the process for $t \geqslant 220 \mathrm{fs}$, a corrugated structure, i.e., a kind of oscillation, is observed in the value $E_{m}^{b i}$. It is considered that such oscillatory dynamics of the value $E_{m}^{b i}$ results from the dynamics of bunched electrons trapped in the deep potential well. The timescale of the oscillation is $\sim 15 \mathrm{fs}$, which is close to that of the inverse bounce frequency $\omega_{b}$ estimated as $T_{b}=2 \pi / \omega_{b} \equiv$ $2 \pi / k \sqrt{m_{e} / e \Phi} \sim 12 \mathrm{fs}$ at around $y=3.1 \mu \mathrm{m}$ in Fig. 8(a3). Similar oscillatory dynamics due to bunched electrons trapped in a potential well is observed in Refs. [46,47].

All these processes happen after the passage of the laser pulse as seen in Fig. 5(b), indicating that the free energy delivered to cluster electrons by the laser pulse on a short timescale $(0 \leqslant t \leqslant 120 \mathrm{fs})$ causes a long timescale ion dynamics ( $t \geqslant 320 \mathrm{fs}$ ) coupled with the evolution of the field $E_{y}$, i.e., the ambipolar field and the bipolar one. Namely, the evolution dynamics including the development and oscillation of the value $E_{m}^{b i}$ during $120 \leqslant t<320 \mathrm{fs}$ [see Fig. 5(b)] is regarded as a transient process in which the system reaches a quasi-steady-state, which evolves with long timescale ion dynamics after the process of energy input in the system by the laser. In the following, we study the process in detail.

Establishment of kinetic equilibrium. Here, the phase space distribution of electrons that evolve in such electric fields and corresponding potentials is investigated. As shown in Fig. 8(b3), at $t=300 \mathrm{fs,} \mathrm{four} \mathrm{types} \mathrm{of} \mathrm{electron} \mathrm{trajectories}$ exist, i.e., (I) that formed by low energy electrons trapped by the shallow well at around $0 \leqslant y \leqslant 1.6 \mu \mathrm{m}$, (II) that by low energy electrons trapped by the deep well at around $y=2.9 \mu \mathrm{m}$, (III) that by relatively high energy electrons passing through the small hill around $y=1.6 \mu \mathrm{m}$ and those trapped by the global potential well ranging from $y=0$ to $y=5 \mu \mathrm{m}$, and (IV) that by high energy electrons passing through two potential hills at around $y=1.6 \mu \mathrm{m}$ and $y=$ $5 \mu \mathrm{m}$. The motions of these different types of electrons in the potential seen in Fig. 8(a3) are considered to sustain the intensity and the structure of the bipolar field on a long timescale as seen in Fig. 5(b). Namely, we interpret the situation shown in Figs. 8(a3) and 8(b3) as an establishment of a quasistationary kinetic equilibrium, which is considered to be related to that of the Bernstein-Greene-Kruskal (BGK) type [48,49].

It is noted that the value $E_{m}^{b i}$ shows a sudden increase at around $t=290 \mathrm{fs}$ as seen in Fig. 5(b). This is explained as follows: In Fig. 9, we show the same physical quantities as Fig. 8 at $t=287,300$, and 320 fs. As seen in Figs. 9(a1) and 9 (a2), due to the decrease of the potential height around $5 \leqslant y \leqslant 7 \mu \mathrm{m}$, which results from the cluster expansion and associated decrease of the ambipolar field, some of the electrons trapped in the global potential well $(0 \leqslant y \leqslant 5 \mu \mathrm{m})$ tend to become passing electrons [see Figs. 9(b1) and 9(b2)]. Then, they lose some of the kinetic energy when they pass through the potential hill $(y \geqslant 5 \mu \mathrm{m})$, and transfer the energy to the bipolar field. Through such dynamics, the bipolar field becomes a quasi-steady-state without changing the topology in the phase space as seen in Figs. 9(a2) and 9(a3). The sudden change of the potential structure is considered to be the process in which the system evolves toward another kinetic equilibrium that is more stable. The lifetime of such a BGK-type quasistationary kinetic equilibrium in the phase space is an important factor that determines the long timescale dynamics of the system, which is one of our future subjects.

\section{THE EFFECT OF THE AMBIENT GAS DENSITY ON THE TRANSITION DYNAMICS OF THE BOUNDARY LAYER}

Here, we investigate the structure and dynamics of the boundary layer for different ambient gas densities in 

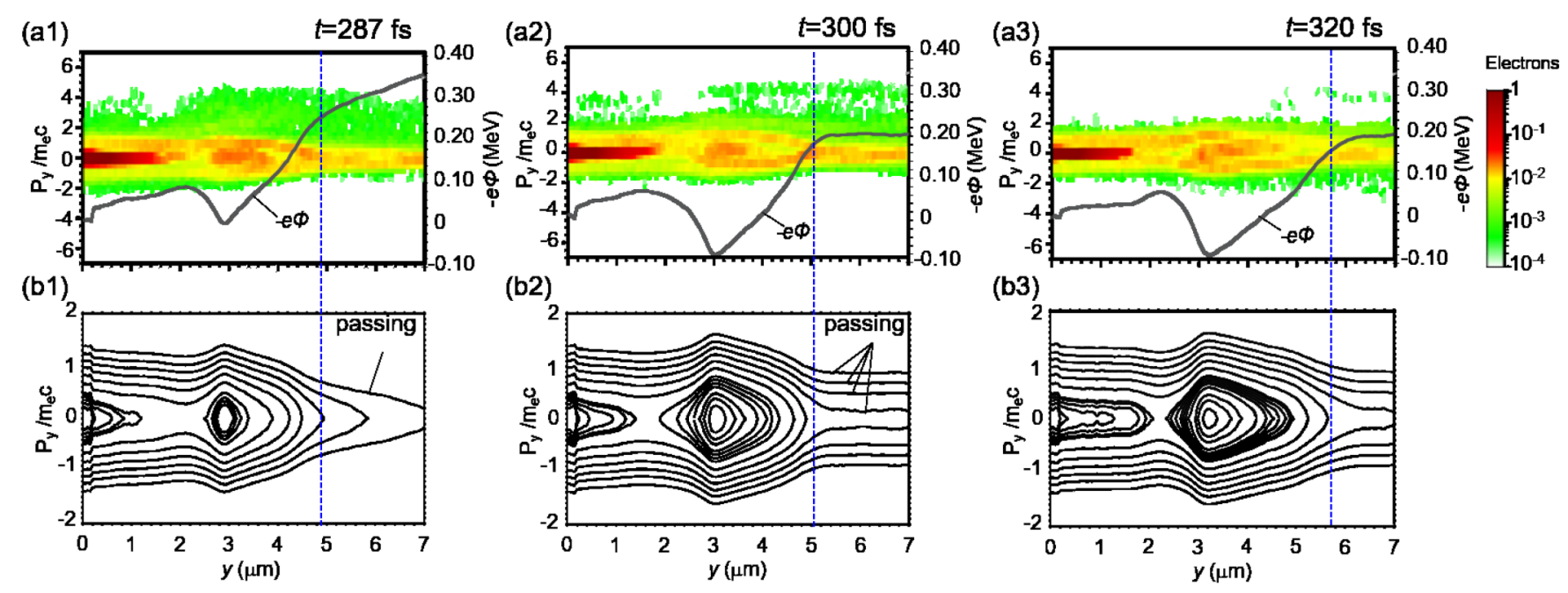

FIG. 9. The 1D cross sectional views for (a1)-(a3) the phase space distributions of electrons and the electrostatic potential for electrons $-e \Phi$ (gray solid line), and (b1)-(b3) the contour plots of electron trajectories along the $+y$ direction from the center of the cluster at (a1), (b1) $t=287 \mathrm{fs}$, (a2), (b2) $t=300 \mathrm{fs}$, and (a3), (b3) $t=320 \mathrm{fs}$. Blue dashed lines indicate the position of the cluster expansion front. The electrostatic potential is set to zero at $y=0$. Phase space distributions consist of electrons in the region of $y \geqslant 0$ and $-0.15 \leqslant x \leqslant+0.15 \mu \mathrm{m}$, and are normalized by the initial charge density of the cluster electrons and displayed on a log scale.

Sec. IV A. The effects of the ambient gas density and the underlying physics on the transition dynamics of the boundary layer are discussed in Sec. IV B.

\section{A. The structure of the boundary layer for different ambient gas densities}

In order to study the density dependence on the kinetic equilibrium characterized by the bipolar electric field, we perform a series of simulations using different densities of the ambient gas while keeping other parameters the same. Figures 10(a)-10(d) show 1D cross sectional views for the charge densities $\rho_{i}^{c l}, \rho_{i}^{a g}, \rho_{e}^{c l}$, and $\rho_{e}^{a g}$ and the field $E_{y}$ along the $+y$ direction at $t=380 \mathrm{fs}$ for different densities of ambient gas electrons $\left(=n_{a g}\right)$, i.e., (a) $0.012 n_{c}$, (b) $0.024 n_{c}$, (c) $0.096 n_{c}$, and (d) $0.192 n_{c}$. Note that case (b) is the same as that in Fig. 3 while the time is different, i.e., $t=300 \mathrm{fs}$ in Fig. 3. When the density $n_{a g}$ becomes higher, more electrons of the ambient gas flow into the cluster expansion front, which weakens the intensity of the ambipolar electric field [see Fig. 14(b)]. As a result, the velocity of the cluster expansion front $v_{f}^{c l}$ decreases, so that the radial location $r_{f}^{c l}$ becomes small as seen in Figs. 10(a)-10(d). On the other hand, the peak charge density around the compressed surface of the ambient gas ions $\rho_{i, f(B)}^{a g}$ increases roughly in proportion to the density $n_{a g}$ as seen in Figs. 10(a)-10(d). As a result, the maximum negative value of the bipolar electric field $E_{m}^{b i}$ around the compressed surface also becomes large in proportion to the density $n_{a g}$ as seen in Figs. 10(a)-10(d).

It is noted that though the velocity $v_{f}^{c l}$ and the value $E_{m}^{b i}$ are different depending on the density $n_{a g}$, the bipolar field shows a structure similar to that of the original case $\left[n_{a g}=0.024 n_{c}\right.$; Fig. 10(b)], which is also sustained. Figure 10(e) shows the phase space distribution of ions in the case of the higher density, $n_{a g}=0.192 n_{c}$. In this case, the propagation of the rarefaction wave in both $\pm y$ directions, i.e., the region of $0.8 \leqslant y \leqslant 2.8 \mu \mathrm{m}$ in the ambient gas, is clearly observed.
Such a propagation also can be seen in Fig. 6(c3) in the case of the density $n_{a g}=0.024 n_{c}$. On the other hand, a dip structure [marked by $(*)$ ] in the phase space distribution of cluster ions is observed at around $y=2.5 \mu \mathrm{m}$, which corresponds to the staircase structure in the charge density distribution of cluster ions seen in Fig. 10(d). The staircase structure is formed due to the deceleration of the cluster ions by the negative component of the bipolar field. The structure is also formed in the case of the density $n_{a g}=0.096 n_{c}$ [see Fig. 10(c)], while hardly seen in the case of the lower densities $n_{a g}=0.012 n_{c}$ and $0.024 n_{c}$ [see Figs. 10(a) and 10(b)].

Figure 11 shows the time evolution of 1D cross sectional views for the charge densities $\rho_{i}^{c l}$ and $\rho_{i}^{a g}$ at various times for the density $n_{a g}=0.192 n_{c}$. As seen in Fig. 11, the sharp peaking structure of the compressed surface starts to collapse as the rarefaction wave from $t \sim 200 \mathrm{fs}$, leading to the formation of the region with a gentle gradient, which shows a similar tendency to that in Fig. 3(e). It is interesting to note that the front of the ambient gas ions, which is located at around $y=1.3 \mu \mathrm{m}(t=200 \mathrm{fs})$, moves in the $-y$ direction after $t \sim 200 \mathrm{fs}$, so that a fixed point can be seen at around $y=1.3 \mu \mathrm{m}$, as seen in Fig. 11 .

Figure 12 shows temporal evolutions for the locations (A) $r_{f}^{c l}$, (B) $r_{f(B)}^{a g}$, and (D) $r_{f(D)}^{a g}$ for the density $n_{a g}=0.192 n_{c}$. The location of the staircase structure of the cluster ions (C) $r_{s}^{c l}$ is also shown. Here, the crossing time $t_{c}$ and the trigger time $t_{r}$ are estimated approximately as $t_{c} \simeq 140 \mathrm{fs}$ and $t_{r} \simeq$ $200 \mathrm{fs}$, respectively, where the times $t_{c}$ and $t_{r}$ are introduced in Fig. 5(a).

As seen in Fig. 12, temporal evolutions for the locations $r_{f}^{c l}$ and $r_{f(B)}^{a g}$ show a similar tendency to those in Fig. 5(a). In this case, the region represented by $r_{f}^{c l(v)}-r_{f}^{c l}$ (indicated by the double-headed black arrow in Fig. 12) becomes wider compared with that for the density $n_{a g}=0.024 n_{c}$ [see Fig. 5(a)], indicating that the velocity $v_{f}^{c l}$ decreases when the density $n_{a g}$ becomes higher as discussed in Figs. 10(a)-10(d). Here, 



FIG. 10. (a)-(d) The 1D cross sectional views for charge density distributions of cluster ions $\rho_{i}^{c l}$ (black solid line), ambient gas ions $\rho_{i}^{a g}$ (blue solid line), cluster electrons $\rho_{e}^{c l}$ (black dotted line), ambient gas electrons $\rho_{e}^{a g}$ (blue dotted line), and the field $E_{y}$ (red solid line) along the $+y$ direction from the center of the cluster at $t=$ $380 \mathrm{fs}$ with the ambient gas density of (a) $0.012 n_{c}$, (b) $0.024 n_{c}$, (c) $0.096 n_{c}$, and (d) $0.192 n_{c}$. Densities are normalized by the initial charge density of the cluster ions. (e) The phase space distributions of ions (cluster and ambient gas) with the ambient gas density of $0.192 n_{c}$. The phase space distributions consist of ions in the region of $y \geqslant 0$ and $-0.15 \leqslant x \leqslant+0.15 \mu \mathrm{m}$, and are normalized by the initial charge density of the cluster ions and displayed on a log scale.



FIG. 11. The time evolution of the $1 \mathrm{D}$ cross sectional views for charge density distributions of ambient gas ions $\rho_{i}^{a g}$ (blue lines) and cluster ions $\rho_{i}^{c l}$ (black lines) at the density $n_{a g}=0.192 n_{c}$ along the $+y$ direction from the center of the cluster at various times. The initial density distributions of the ambient gas (blue dotted line) and cluster (black dotted line) ions are also shown. Densities are normalized by the initial charge density of the ambient gas or cluster ions.

the location $r_{f}^{c l(v)}$ represents the cluster expansion front in the vacuum as discussed in Fig. 5(a). The location $r_{f(B)}^{a g}$ exhibiting the peak density propagates in the $+y$ direction. On the other hand, the location $r_{f(D)}^{a g}$ exhibiting the front propagates initially in the $+y$ direction $\left(t<t_{r}\right)$ while finally in the $-y$ direction $\left(t \geqslant t_{r}\right)$, resulting in the contamination of the cluster core by ambient gas ions [see also Fig. 11].

Here, in order to investigate the relation between the formation of staircase structure in cluster ions and the destruction of the compressed surface of ambient gas ions, we show 2D images for the charge densities $\rho_{i}^{c l}$ and $\rho_{i}^{a g}$, respectively, in Fig. 13. As clearly seen in Figs. 13(c) and 13(d), the front of the rarefaction wave radially propagates inside the cluster. Simultaneously, the staircase structure, which corresponds to the ring structure in the cluster charge density distribution indicated by the arrow in Fig. 13(a), further evolves, leading to the density hump as seen in Fig. 13(b). That is, the formation of structure in the cluster ions and the destruction of structure in the ambient gas ions as the rarefaction wave occur in pairs. It is worthwhile to consider the relationship between such ring structures observed in the present simulation and those observed in the universe, e.g., rings after supernova explosions [39].

These results indicate that the kinetic equilibrium characterized by the bipolar field structure also exists in high density cases [see Figs. 10(c) and 10(d)], while the compressed surface in high density cases evolves into the rarefaction wave with a fast timescale compared with that in low density cases. The timescale on which the structures associated with ion dynamics evolve into the rarefaction wave is much slower than that of the motion of electrons $\left(T_{b} \sim 12 \mathrm{fs}\right.$; see Sec. IIIC). That is, such an equilibrium state is considered to be a 


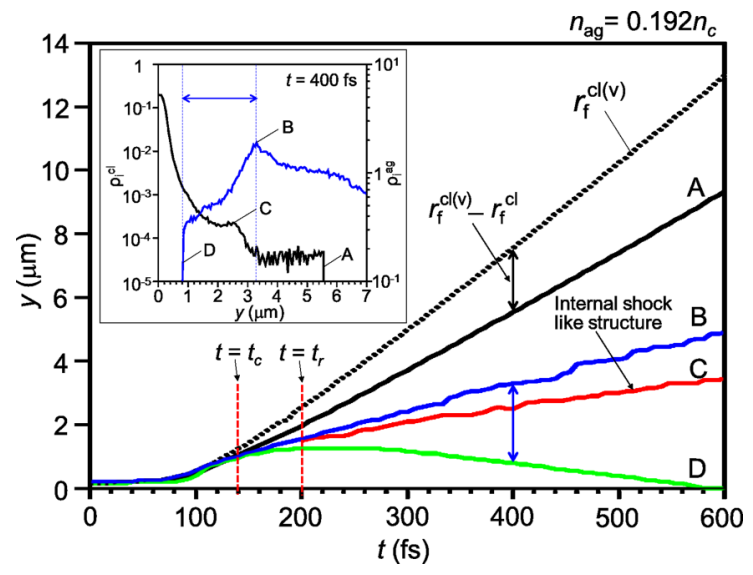

FIG. 12. Temporal evolutions for the location of the expansion front $r_{f}^{c l}$ (black solid line, A) and that of the staircase structure $r_{s}^{c l}$ (red solid line, C) of the cluster ions, and that exhibiting the peak density $r_{f(B)}^{a g}$ (blue solid line, B) and that exhibiting the front $r_{f(D)}^{a g}$ (green solid line, D) of ambient gas ions along the $+y$ direction from the center of the cluster at the density $n_{a g}=0.192 n_{c}$. The inset shows a $1 \mathrm{D}$ cross sectional view for the density distributions of ambient gas ions (blue solid line) and cluster ions (black solid line) at $t=400 \mathrm{fs}$. The locations A, B, C, and D in the inset correspond to those in the figure. The black dotted line represents the temporal evolution for the location of the expansion front of the cluster ions in a vacuum $r_{f}^{c l(v)}$ as a reference.

common physics process, which is formed at the boundary layer during the interaction between two distinct collisionless plasmas.



(c)



(d)



FIG. 13. Snapshots of the 2D images for charge density distributions of (a), (b) the cluster ions $\rho_{i}^{c l}$ and (c), (d) the ambient gas ions $\rho_{i}^{a g}$ at (a), (c) $t=400 \mathrm{fs}$ and (b), (d) $600 \mathrm{fs}$ at the density $n_{a g}=0.192 n_{c}$. Densities are normalized by the initial charge density of the cluster ions and displayed on a log scale.


FIG. 14. (a) The time $t=t_{c}$ at which the crossing between the cluster expansion front and the compressed surface of ambient gas ions takes place (the red solid line) and the time $t=t_{r}$ at which the rarefaction wave is triggered (the red dashed line) at the density $n_{a g}=0.012 n_{c}, 0.024 n_{c}, 0.048 n_{c}, 0.096 n_{c}$, and $0.192 n_{c}$, respectively. The time $t_{c}^{(m)}$ represents the crossing time derived by using the function of the electric field modeled as Eq. (4) from the viewpoint of particle motions in the laboratory frame (red dotted line). The maximum energy of ambient gas ions $W_{m}^{a g}$ at $t=400$ fs is also shown (blue solid line). (b) The 1D cross sectional views for the ambipolar electric field $E_{y}$ for the different ambient gas density $n_{a g}$ along the $+y$ direction from the center of the cluster at $t=120 \mathrm{fs}$. The field $E_{y}$ outside the cluster is approximately fitted by the exponential functions with different amplitude $E_{0}$ and the scale length $L_{e}$, which is represented by the black dashed line.

\section{B. Density dependence of transition times}

As discussed in Sec. III A, the dynamics of the boundary layer evolves in three successive phases characterized by typical transition times, which are defined in Fig. 5(a), i.e., the crossing time $t_{c}$ and the trigger time $t_{r}$. In this subsection, we discuss how the initial ambient gas density $n_{a g}$ affects these transition times.

Figure 14(a) shows the times $t_{c}$ and $t_{r}$ as a function of the density $n_{a g}$. The maximum energy of ambient gas ions $W_{m}^{a g}$ at $t=400 \mathrm{fs}$ is also shown. The crossing time $t_{c}$ is found to weakly depend on the density $n_{a g}$, which is explained as follows. As discussed in Sec. III B (under acceleration by nonlinear wave), the contact surface disappears when the upstream ambient gas ions pass over the shock potential 
associated with the ambipolar electric field, leading to the crossing [see Fig. 4(d1)].

Figure 14(b) shows the spatial distribution of the ambipolar electric field $E_{y}$ for the different densities $n_{a g}$ at $t=120 \mathrm{fs}$. It is found that the amplitude of the field $E_{y}$ decreases as the density $n_{a g}$ increases. This is because the shielding of the field $E_{y}$ becomes more effective for higher ambient gas density. On the other hand, it is interesting to note that the field $E_{y}$ outside the cluster exhibits spatial distributions approximately fitted by an exponential function not only in the vacuum case but also in the case with ambient gas as follows:

$$
\tilde{E}(y)=E_{0} \exp \left(-\frac{y-r_{f}^{c l}}{L_{e}}\right),
$$

where $E_{0}$ is the amplitude of the field at the cluster expansion front $\left(y=r_{f}^{c l}\right)$ and $L_{e}$ is the scale length of the field. Here, as seen in Fig. 14(b), as the density $n_{a g}$ increases, the amplitude $E_{0}$ becomes smaller and the scale length $L_{e}$ becomes shorter, while keeping the exponential function form. This result suggests that the spatial distribution of the field $E_{y}$ suffers from a constraint, i.e., the exponential form, during the evolution in phase 1. In Appendix A, we study this feature by solving the Poisson equation in the case without ambient gas, i.e., the case of vacuum in Fig. 14(b), and discuss the background.

As the density $n_{a g}$ increases, the amplitude of the field $E_{y}$ at the cluster expansion front $\left(y=r_{f}^{c l}\right)$ decreases. Then, the shock velocity $v_{f}^{c l}$ in Eq. (2) becomes small. As a result, the kinetic energy of upstream ambient gas ions $K_{1}$ in Eq. (2) also becomes small, which is the factor to prolong the crossing time $t_{c}$. On the other hand, as the density $n_{a g}$ increases, not only the field $E_{y}$ becomes weaker but also the scale length $\left|\partial\left(\ln E_{y}\right) / \partial y\right|^{-1}$ around the upstream region for the shock front $r_{f}^{c l}$, i.e., $y>r_{f}^{c l}$, becomes shorter [see Fig. 14(b)]. As a result, the height of the shock potential calculated by

$$
e \Phi_{\max } \simeq \int_{r_{b}}^{r_{f}^{c l}}\left(-e E_{y}\right) d y
$$

also becomes small, which is the factor to shorten the crossing time $t_{c}$. Here, the location $r_{b}$ represents that of the boundary between the ambient gas and the vacuum in the $+y$ direction, i.e., $r_{b}=7.68 \mu \mathrm{m}$. These two opposite effects are considered to keep the crossing time $t_{c}$ almost constant for the increase of the density $n_{a g}$. The time $t_{c}^{(m)}$ in Fig. 14(a) represents the crossing time derived by using the function of the electric field modeled as Eq. (4) from the viewpoint of particle motions in the laboratory frame, which we discuss in Appendix B.

On the other hand, the trigger time $t_{r}$ becomes short as the density $n_{a g}$ increases, which is explained as follows. The propagation velocity of the location exhibiting the peak density of ambient gas ions $v_{f(B)}^{a g}$ in Eq. (3) weakly depends on the density $n_{a g}$. Namely, the kinetic energy $K_{2}$ in Eq. (3) also weakly depends on the density $n_{a g}$, which is the factor to keep the trigger time $t_{r}$. On the other hand, as the density $n_{a g}$ increases, the field $E_{y}$ becomes weak and the length scale of the field $E_{y}$ around the nonlinear wave becomes short. Accordingly, the height of the electrostatic potential calculated by

$$
e \Phi_{\max } \simeq \int_{r_{b}}^{r_{f(B)}^{a g}}\left(-e E_{y}\right) d y
$$

also becomes small. As a result, in high density cases, upstream ambient gas ions can pass over the potential at an earlier time than that in low density cases, which shortens the trigger time $t_{r}$.

Finally, the maximum kinetic energy of ambient gas ions $W_{m}^{a g}$ becomes small as the density $n_{a g}$ increases. The ambient gas ions having the maximum velocity originate from the early-phase shock acceleration. Namely, $W_{m}^{a g}$ is strongly affected by the shock velocity $v_{f}^{c l}$ which becomes small in high density cases.

\section{CONCLUSION}

We have investigated the dynamics of the boundary layer in between two distinct collisionless plasmas, created by the expansion of the femtosecond laser-irradiated cluster in an ambient gas, and the associated acceleration of the ambient gas ions with the 2D PIC simulations using EPIC3D. We found that the dynamics of the boundary layer is classified into three successive phases, i.e., phase 1: cluster expansion with keeping the contact surface between the cluster expansion front and the compressed surface of ambient gas ions, phase 2: the formation of an overlapping region between them, and phase 3: the collapse of the sharp peaking structure of compressed surface as the rarefaction wave.

In phase 1 (before $t \sim 150 \mathrm{fs}$ ), the contact surface exists during the expansion of the cluster ions and the compression of the ambient gas ions. In this phase, the ambient gas ions are reflected by the electrostatic potential associated with the ambipolar electric field around the contact surface, which causes the collisionless shock acceleration. In phase $2(150<t<250 \mathrm{fs})$, the contact surface disappears and the overlapping region between the cluster expansion front and the compressed surface is formed. In this phase, in addition to the ambipolar electric field around the cluster expansion front, a bipolar electric field is established around the compressed surface. The compressed surface propagates as the nonlinear wave, which causes an acceleration of ambient gas ions similar to that by the shock. In phase 3 (after $t \sim$ $250 \mathrm{fs}$ ), the compressed surface evolves as a rarefaction wave, which coincides with the fact that some of the ambient gas ions change their orbits from reflection to passing across the potential hill.

We found that the peak value of the ambipolar field decays quickly, while that of the bipolar field persists for a long time even after the passage of the laser pulse. This is because the nonlinear wave structure associated with the bipolar field is sustained as a quasistationary kinetic equilibrium established via the balance between trapped and passing electrons on the electrostatic potential. Namely, through the transient process between the successive three phases in the above, the system reaches a quasi-steady-state, which evolves with long timescale ion dynamics after the short timescale electron dynamics caused by the laser input. The equilibrium we reported in this paper is considered to be related 
to Bernstein-Greene-Kruskal (BGK) type kinetic equilibrium [48,49].

We studied the dynamics in a wide range of ambient gas densities. We found that various dynamics, e.g., the electric field causing shock acceleration, timings in which the crossing and rarefaction waves are triggered, etc., depend on the ambient gas density. However, the quasistationary kinetic equilibrium dominated by fast electrons is established not only in lower but also in higher density cases. The formation of such an equilibrium has been reported in space plasmas such as the plasma sheet boundary layer in the Earth's magnetotail [50] and the downward-current regions of the auroral zone [51]. The future observation of such nonlinear waves under laboratory conditions may help understand behaviors of nonlinear electrostatic waves and related particle accelerations in space and astrophysical plasmas.

On the other hand, in high density cases, the ringlike and/or staircase structure in the cluster ions is clearly observed during the destruction process of the compressed surface of the ambient gas. Such ringlike and/or staircase structures are found to be widely observed in space and astrophysical plasmas. It is interesting to study the similarity with the present case. In the future, we will study the long timescale $(\sim$ ps $)$ dynamics.

Here, we consider the $2 \mathrm{D}$ case while it is valuable to discuss the effect of the dimension of the system on the dynamics and structure, specifically on the formation of the kinetic equilibrium. Since the phenomena after the laser irradiation is approximately spherically symmetric, 1D simulation assuming electron distribution functions is possible, which would have an advantage in parametrizing the phenomena and/or deriving scaling law. This is a future work. On the other hand, in terms of the 3D effect, the establishment of the kinetic equilibrium observed in the 2D simulation is also expected to take place because it is driven by the 1D motion of electrons in the radial direction of the cluster. However, since the electrostatic potential energy contained in a cluster constituted only by ions and also that per ion is different between $2 \mathrm{D}$ and 3D cases, 2D simulations do not fully reproduce the dynamics of 3D spherical cluster. In the future, we will perform 3D simulations and confirm the adequacy of the present $2 \mathrm{D}$ simulations. Moreover, it is worthwhile to study the critical condition for the formation of kinetic equilibrium for other parameters such as cluster size and laser intensity, which is a coming work.

\section{ACKNOWLEDGMENTS}

This work was supported by Grant-in-Aids for Scientific Research No. 25287153, No. 17H01180, and No. JP19H00668 by JSPS KAKENHI. We thank Dr. Kenji Imadera, Dr. Domenico Doria, Dr. Lorenzo Romagnani, and Professor Youichi Sakawa for fruitful discussions about this work.

\section{APPENDIX A}

\section{The model of ambipolar electric field and numerical scheme}

Here, we discuss the origin exhibiting an exponential function form of the ambipolar electric field $E_{y}$ produced around

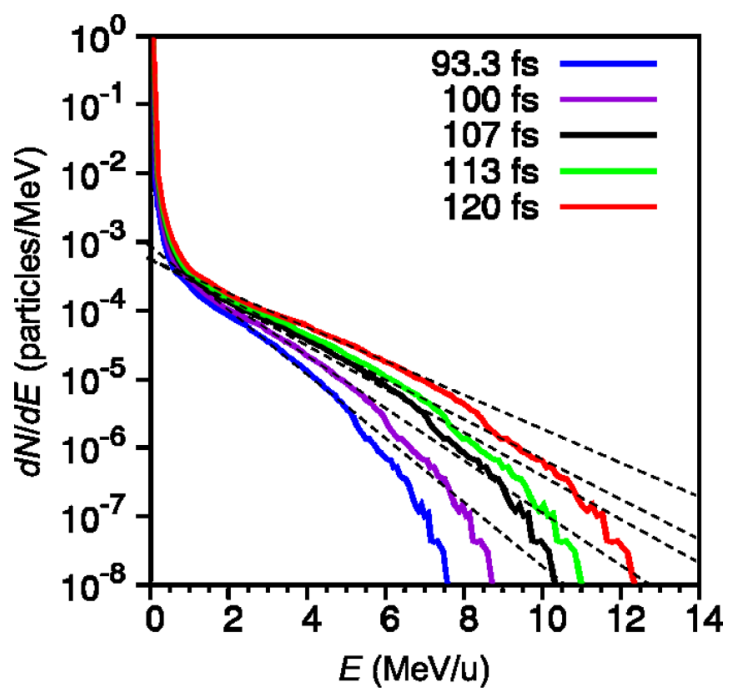

FIG. 15. Energy distributions of the cluster electrons at $t=$ $93.3 \mathrm{fs}, 100 \mathrm{fs}, 107 \mathrm{fs}, 113 \mathrm{fs}$, and $120 \mathrm{fs}$, respectively. Black dashed lines show the exponential function.

the cluster expansion front seen in Fig. 14(b) using a kinetic model of isothermal plasma expansion into vacuum.

In Fig. 15, we show the energy distribution of cluster electrons at different times in the early stage of the cluster expansion in the case of the vacuum in Fig. 14(b) (green line). Here, it can be seen that each distribution besides the low energy bulk region is fitted by a Maxwellian whose temperature increases with time (black dashed lines in Fig. 15) and is truncated, exhibiting a maximum energy. This feature is widely observed as in the present case in which the interaction is limited to a short time of laser irradiation on matter. A similar distribution is considered to take place for an oblique incident of laser on a target, where the heating region is spatially localized around the resonant surface.

Motivated by these characteristics of electron energy distribution, here we consider one-dimensional plasma expansion into vacuum in a nonrelativistic regime using the kinetic model given in Ref. [5] to understand the qualitative nature of the spatial distribution of ambipolar electric field $E_{y}$ discussed in the above. Here, we consider an ion density distribution given by $n_{i}(y)$ for $0 \leqslant y \leqslant r_{f}$, where $r_{f}$ is the location of the ion expansion front. The ion charge state $Z=1$ is assumed. We employ a BGK-type electron distribution function with a constant electron temperature $T_{e}$ under the condition that the velocity distribution is truncated at $\left|v_{y}\right|=v_{0}\left(-v_{0} \leqslant v_{y} \leqslant v_{0}\right)$ with the maximum energy $1 / 2 m_{e} v_{0}^{2}\left(=\varepsilon_{\max }\right)$, which is similar to that seen in Fig. 15. Then, the corresponding electron distribution function is represented by

$$
f\left(y, v_{y}\right)= \begin{cases}n_{b}\left(\frac{m_{e}}{2 \pi T_{e}}\right)^{\frac{3}{2}} \exp \left(-\frac{m_{e}}{2 T_{e}} v_{y}^{2}+\frac{e \Phi}{T_{e}}\right), & \left(\left|v_{y}\right| \leqslant v_{0}\right), \\ 0, & \left(\left|v_{y}\right|>v_{0}\right),\end{cases}
$$

where $\Phi$ is the electrostatic potential, and $n_{b}$ is the normalization factor related to the electron density. Here, $n_{b}$ is determined as an eigenvalue so as to conserve the total number of electrons in the system. 
By integrating Eq. (A1) for $-v_{0} \leqslant v_{y} \leqslant v_{0}$, we obtain the local electron density, which leads to the following Poisson equations:

for $0 \leqslant \tilde{y} \leqslant 1$,

$$
\frac{d^{2} \tilde{\Phi}}{d \tilde{y}^{2}}=\frac{1}{{\tilde{\lambda_{D}}}^{2}}\left[\tilde{n_{b}} \exp (\tilde{\Phi}) \operatorname{erf}(\sqrt{\tilde{\Phi}})-\tilde{n}_{i}(\tilde{y})\right] ;
$$

for $1 \leqslant \tilde{y} \leqslant \tilde{r}_{e}$,

$$
\frac{d^{2} \tilde{\Phi}}{d \tilde{y}^{2}}=\frac{\tilde{n_{b}}}{{\tilde{\lambda_{D}}}^{2}} \exp (\tilde{\Phi}) \operatorname{erf}(\sqrt{\tilde{\Phi}}) ;
$$

where $\operatorname{erf}(x)$ is the error function. Normalizations are given by $\tilde{y}=y / r_{f}, \tilde{\Phi}=e \Phi / T_{e}, \tilde{\lambda_{D}}=\lambda_{D} / r_{f}, \tilde{n_{b}}=n_{b} / n_{0}$, and $\tilde{n}_{i}(\tilde{y})=$ $n_{i}(\tilde{y}) / n_{0}$, respectively, where $\lambda_{D}=\sqrt{T_{e} / 4 \pi n_{0} e^{2}}$ is the Debye length and $n_{0}$ is the initial ion density. Here, we define the electron temperature $T_{e 0}=4 \pi n_{0} e^{2} r_{f}^{2}$, where the corresponding Debye length is equal to the cluster radius, i.e., $\sqrt{T_{e 0} / 4 \pi n_{0} e^{2}}=r_{f}$. We use the temperature as the normalization of the electron temperature, i.e., $\tilde{T}_{e}=T_{e} / T_{e 0}$, which leads to $\tilde{\lambda_{D}}=\sqrt{\tilde{T}_{e}}$.

By performing energy integration to Eq. (A3) under the boundary conditions of

$$
\tilde{\Phi}\left(\tilde{y}=\tilde{r}_{e}\right)=0 \text { and }\left(\frac{d \tilde{\Phi}}{d \tilde{y}}\right)_{\tilde{y}=\tilde{r}_{e}}=0,
$$

we obtain the following relation for the potential $\tilde{\Phi}$ in the region $1 \leqslant \tilde{y} \leqslant \tilde{r}_{e}\left(\tilde{r}_{e}\right.$ : the location of the electron front normalized by $r_{f}$ ),

$$
\frac{1}{2}\left(\frac{d \tilde{\Phi}}{d \tilde{y}}\right)^{2}=\frac{\tilde{n_{b}}}{{\tilde{\lambda_{D}}}^{2}}\left[\exp (\tilde{\Phi}) \operatorname{erf}(\sqrt{\tilde{\Phi}})-2 \sqrt{\frac{\tilde{\Phi}}{\pi}}\right] .
$$

We solve Eq. (A2) as an eigenvalue problem based on Ref. [5] under the boundary conditions at $\tilde{y}=0$, i.e., $\tilde{\Phi}=$ $\tilde{\varepsilon}_{\max }\left(\equiv \varepsilon_{\max } / T_{e}\right)$ and $d \tilde{\Phi} / d \tilde{y}=0$, and those at $\tilde{y}=1$, i.e., trial values of $\tilde{\Phi}$ and $d \tilde{\Phi} / d \tilde{y}$, which are eigenvalues together with the density $\tilde{n_{b}}$. Here, we choose a radial location $\tilde{y}=b$ between $\tilde{y}=0$ and $\tilde{y}=1(0<b<1)$ and integrate Eq. (A2) from both boundaries at $\tilde{y}=0$ and $\tilde{y}=1$ toward $\tilde{y}=b$. Here, we simply choose $b=1 / 2$ in the present case. A mismatch of the potential $\tilde{\Phi}$ and the derivative $d \tilde{\Phi} / d \tilde{y}$ takes place at $\tilde{y}=b$ for solutions solved from $\tilde{y}=0$, i.e., $\tilde{\Phi}^{(-)}$and $d \tilde{\Phi}^{(-)} / d \tilde{y}$, and those from $\tilde{y}=1$, i.e., $\tilde{\Phi}^{(+)}$and $d \tilde{\Phi}^{(+)} / d \tilde{y}$. The eigenvalues are then corrected so that the solutions at $\tilde{y}=b$ solved from both sides become continuous and then the mismatch vanishes.

The procedure is repeated so that the eigenvalues are converged with a high accuracy, e.g., $10^{-8}$ in the relative value. To ensure a quadratic convergence for true eigenvalues during the repetition of the above shooting scheme, we introduce a Newton algorithm in which independent linearized equations with the inhomogeneous term for Eq. (A2) for the potential $\tilde{\Phi}$ are incorporated with proper boundary conditions, which follows the procedure introduced in Ref. [52].

In the region outside the ion front, i.e., $1 \leqslant \tilde{y} \leqslant \tilde{r}_{e}$, the potential $\tilde{\Phi}$ is determined by integrating Eq. (A5) from $\tilde{y}=$ 1 toward the outside of the ion front using the boundary


FIG. 16. (a) The spatial distributions of ion density (black solid line), electron density (black dotted line), and electric field $\tilde{E}_{y}$ (purple solid line) at the initial state, i.e., at $t=0$, in the region $0 \leqslant \tilde{y} \leqslant$ 2 for $\tilde{\varepsilon}_{\max }=7$. The spatial distributions of (b) electric field $\tilde{E}_{y}$ and (c) potential energy $-e \tilde{\Phi}$ for four cases, i.e., $\tilde{\varepsilon}_{\max }=4,5,6$, and 7 . Here, $\tilde{\varepsilon}_{\max }$ represents the maximum electron energy that is normalized by the electron temperature $T_{e}$. The black dashed lines in (b) represent the exponential function for each energy $\tilde{\varepsilon}_{\max }$, and the black arrows in (c) represent the location of the electron front $\tilde{r}_{e}$, which is normalized by $r_{f}$, for each energy $\tilde{\varepsilon}_{\max }$.

condition $\tilde{\Phi}$ at $\tilde{y}=1$, which is given by one of three eigenvalues. Here, the location $\tilde{r}_{e}$ is determined by that satisfying $\tilde{\Phi}=0$. Note that $d \tilde{\Phi} / d \tilde{y}=0$ at $\tilde{y}=\tilde{r}_{e}$ is also satisfied.

\section{Numerical results and analyses}

Here, we consider a simple case in which the ion density distribution is constant with a sharp boundary at the ion front, i.e., $\tilde{n}_{i}(\tilde{y})\left[\equiv n_{i}(\tilde{y}) / n_{0}\right]=1$ for $0 \leqslant \tilde{y} \leqslant 1$ while $\tilde{n}_{i}(\tilde{y})=0$ for $1 \leqslant \tilde{y}$, which corresponds to the initial state in which the expansion starts. The electron temperature $\tilde{T}_{e}\left(\equiv T_{e} / T_{e 0}\right)$ is set to 0.1 , i.e., $100 \mathrm{keV}$. In the present case, we employ the ion density $n_{0}=3.8 \times 10^{23} \mathrm{~cm}^{-3}$, the slab length $r=0.125 \mu \mathrm{m}$ (initial cluster radius), and $T_{e 0}=1 \mathrm{MeV}$, which correspond to the present simulation parameters.

In Fig. 16(a), we show the resulting spatial distributions of electron density (black dotted line) and electric field $\tilde{E}_{y}$ 




FIG. 17. The spatial distributions of the electric field $\tilde{E}_{y}$ (purple solid line) and the potential energy $e \tilde{\Phi}$ (purple dashed line) for $\tilde{\varepsilon}_{\max }=7$. The field $\tilde{E}_{y_{1}}$ (brown solid line), which is calculated by using the first term on the RHS of Eq. (A5), and the field $\tilde{E}_{y_{2}}$ (orange solid line), which is calculated by using the second, are also shown. The black dashed line shows a nearly exponential function in the intermediate radial region except those near the ion front and electron front.

(purple solid line) in the region $0 \leqslant \tilde{y} \leqslant 2$ for $\tilde{\varepsilon}_{\max }=7$. We also show the spatial distribution of the field $\tilde{E}_{y}$ on a $\log$ scale in Fig. 16(b) and that of the potential energy $-e \tilde{\Phi}$ in Fig. 16(c), in the region $0 \leqslant \tilde{y} \leqslant 10$. Different lines in Figs. 16(b) and 16(c) correspond to those for the different maximum electron energy, i.e., $\tilde{\varepsilon}_{\max }=4,5,6$, and 7 , respectively. The location of the electron front for each maximum energy $\tilde{\varepsilon}_{\max }$, i.e., $\tilde{r}_{e}$, is indicated by the arrow in Fig. 16(c). As seen in Fig. 16(a), the ambipolar electric field $\tilde{E}_{y}$ exhibits a peak at the ion front whose width is estimated by the Debye length $\tilde{\lambda_{D}}=\sqrt{\tilde{T}_{e}} \sim 0.32$. It is shown in Figs. 16(b) and $16(\mathrm{c})$ that as the maximum electron energy $\tilde{\varepsilon}_{\max }$ increases, the location of the electron front $\tilde{r}_{e}$ (indicated by the black arrows) shifts in the $+\tilde{y}$ direction, where the boundary conditions of Eq. (A4) are satisfied. It is noted that potential distributions in Fig. 16(c) show a similar tendency to that in Fig. 8(a1), which is the case of the cluster expansion into the ambient gas in the early phase, i.e., phase 1 discussed in Sec. III A. In this phase, the effect of the ambient gas is almost negligible, so that the cluster expansion dynamics into the ambient gas is approximated as that into the vacuum.

Here, it is interesting to note that the radial region of the field $\tilde{E}_{y}$ except that near the ion front $(\tilde{y} \simeq 1)$ and also that near the electron front $\left(\tilde{y} \simeq \tilde{r}_{e}\right)$ is well fitted by the exponential function (black dashed lines), which becomes wider for larger $\tilde{\varepsilon}_{\max }$ as seen in Fig. 16(b). This feature coincides with that observed in Fig. 14(b) and the associated discussion related to Eq. (4).

To study the background of the exponential function form in Fig. 17, we pick up the case of $\tilde{\varepsilon}_{\max }=7$ in Fig. 16 and show the contribution from the first and second terms on the right-hand side (RHS) of Eq. (A5) on the electric field $\tilde{E}_{y}$, which we describe in the following. Namely, the first terms become dominant near the ion front $(\tilde{y} \simeq 1)$, where $\tilde{\Phi} \simeq 6$, while the first term and the second are almost comparable near the electron front $\left(\tilde{y} \simeq \tilde{r}_{e}\right)$, where $\tilde{\Phi} \simeq 0$. In order to show these features, we introduce the fields $\tilde{E}_{y_{1}}$ (brown solid line) and $\tilde{E}_{y_{2}}$ (orange solid line), which are given by using the first and second terms on the RHS of Eq. (A5) as follows,

$$
\begin{gathered}
\tilde{E}_{y_{1}}=\frac{1}{\tilde{\lambda_{D}}} \sqrt{2 \tilde{n_{b}} \exp (\tilde{\Phi}) \operatorname{erf}(\sqrt{\tilde{\Phi}}),} \\
{\tilde{E_{y_{2}}}}=2 \frac{\sqrt{\tilde{n_{b}}}}{\tilde{\lambda_{D}}}\left(\frac{\tilde{\Phi}}{\pi}\right)^{\frac{1}{4}} .
\end{gathered}
$$

In Fig. 17, we show these fields, i.e., $\tilde{E}_{y_{1}}$ by the brown line and $\tilde{E}_{y_{2}}$ by the orange line. Here, it is noted that the potential $\tilde{\Phi}$ used in Eqs. (A6) and (A7) is obtained by integrating Eq. (A5).

In region $\mathrm{I}$ near the ion expansion front, i.e., $\tilde{y} \simeq 1$, the relation $\tilde{E}_{y_{1}} \gg \tilde{E}_{y_{2}}$ is satisfied as seen in Fig. 17. Therefore, Eq. (A5) is approximated as

$$
\frac{1}{2}\left(\frac{d \tilde{\Phi}}{d \tilde{y}}\right)^{2}=\frac{\tilde{n_{b}}}{{\tilde{\lambda_{D}}}^{2}} \exp (\tilde{\Phi}) \operatorname{erf}(\sqrt{\tilde{\Phi}}) .
$$

By applying the asymptotic expansion to the error function $\operatorname{erf}(x)$, i.e., $\operatorname{erf}(\sqrt{\tilde{\Phi}}) \simeq 1-\exp (-\tilde{\Phi}) / \sqrt{\pi \tilde{\Phi}}$, we obtain

$$
\frac{1}{2}\left(\frac{d \tilde{\Phi}}{d \tilde{y}}\right)^{2}=\frac{\tilde{n_{b}}}{{\tilde{\lambda_{D}}}^{2}}\left[\exp (\tilde{\Phi})-\frac{1}{\sqrt{\pi \tilde{\Phi}}}\right] .
$$

Furthermore, by neglecting the second term compared to the first term in Eq. (A9), we obtain the following equation,

$$
\frac{d \tilde{\Phi}}{d \tilde{y}}=\alpha \exp \left(\frac{\tilde{\Phi}}{2}\right),
$$

where $\alpha=\sqrt{2 \tilde{n_{b}}} / \tilde{\lambda_{D}}$. By integrating Eq. (A10) under the boundary value of $\tilde{\Phi}$ at $\tilde{y}=1$, which is the eigenvalue obtained by solving Eq. (A2), we obtain the formula of the field $\tilde{E}_{y}$ near the ion front,

$$
\tilde{E}_{y}=\frac{2}{\tilde{y}-\frac{2}{\alpha} \exp \left[-\frac{\tilde{\Phi}(\tilde{y}=1)}{2}\right]-1} \quad(\tilde{y} \simeq 1) .
$$

The behavior given by Eq. (A11) is consistent with that of the field $\tilde{E}_{y}$, which quickly increases near $\tilde{y}=1$, as seen in Fig. 17 (brown and purple lines).

On the other hand, in region III near the electron front, i.e., $\tilde{y} \simeq \tilde{r}_{e}$, the field $\tilde{E}_{y_{2}}$ becomes comparable to the field $\tilde{E}_{y_{1}}$ as seen in Fig. 17. Therefore, by applying the Taylor expansion around $\tilde{\Phi}=0$ to $\exp (x)$ and $\operatorname{erf}(x)$, i.e., $\exp (\tilde{\Phi}) \simeq 1+\tilde{\Phi}$ and $\operatorname{erf}(\sqrt{\tilde{\Phi}}) \simeq 2 \sqrt{\tilde{\Phi} / \pi}$, respectively, one obtains

$$
\begin{gathered}
\frac{1}{2}\left(\frac{d \tilde{\Phi}}{d \tilde{y}}\right)^{2}=\frac{2}{\sqrt{\pi}} \frac{\tilde{n_{b}}}{{\tilde{\lambda_{D}}}^{2}} \tilde{\Phi}^{\frac{3}{2}}, \\
\therefore \frac{d \tilde{\Phi}}{d \tilde{y}}=\beta \tilde{\Phi}^{\frac{3}{4}},
\end{gathered}
$$

where $\beta=\sqrt{(4 / \sqrt{\pi}) \tilde{n_{b}}} / \tilde{\lambda_{D}}$. By integrating Eq. (A13) under the boundary value of $\tilde{\Phi}=0$ at $\tilde{y}=\tilde{r}_{e}$ [Eq. (A4)], we obtain the formula of the field $\tilde{E}_{y}$ around the electron front as follows,

$$
\tilde{E}_{y}=-\frac{\beta^{4}\left(\tilde{y}-\tilde{r}_{e}\right)^{3}}{64} \quad\left(\tilde{y} \simeq \tilde{r}_{e}\right)
$$

The behavior given by Eq. (A14) is also consistent with that of the field $\tilde{E}_{y}$, which quickly damps near $\tilde{y}=\tilde{r}_{e}$ as seen in Fig. 17 (brown and orange lines). Namely, the intermediate 

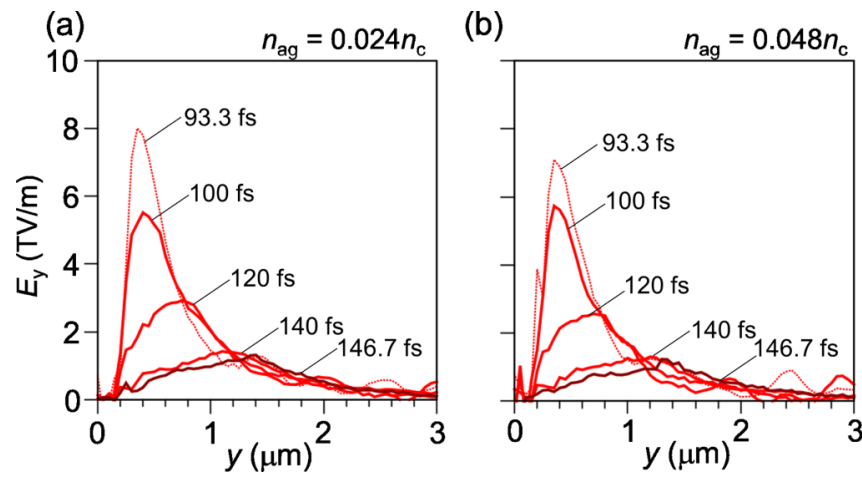

FIG. 18. The spatial distribution of the field $E_{y}$ at different times for the density (a) $n_{a g}=0.024 n_{c}$ and (b) $n_{a g}=0.048 n_{c}$.

region between regions I and III, i.e., region II, exhibiting an exponential function, is regarded as an inertia and/or adiabatic region that connects two regions, i.e., regions I and II, where electrons suffer from strong acceleration and deceleration due to the significant change of electron orbits near the ion expansion front and near the electron front, respectively.

In the present case, we treat the electron dynamics in a nonrelativistic regime because the effective electron temperature is approximated as $T_{e} \sim 100 \mathrm{keV}$ in the early phase of the cluster expansion into the vacuum (see Fig. 15). On the other hand, even under this condition, i.e., $T_{e} \sim 100 \mathrm{keV}$, the dynamics for $\tilde{\varepsilon}_{\max } \geqslant 5\left(\varepsilon_{\max } \geqslant 5 T_{e}\right)$ becomes relativistic. Namely, we need to treat the relativistic Maxwellian distribution for electrons and solve the corresponding Poisson equation. Furthermore, here, we consider a simple slab geometry assuming that the effect of the cylindrical geometry is small. However, the effect is important especially in the early phase of expansion. In order to prove the feature of the exponential function form of the field $E_{r}$ observed in the simulation correctly, we need to include such effects consistently, which remains as a future work.

\section{APPENDIX B: DERIVATION OF THE CROSSING TIME}

Here, we derive the crossing time by using the function of the electric field modeled as Eq. (4) from the viewpoint of particle motions in the laboratory frame.

In Fig. 18, we show the spatial distribution of the field $E_{y}$, which is obtained from the simulations, at different times for the densities $n_{a g}=$ (a) $0.024 n_{c}$ and (b) $0.048 n_{c}$. The fields $E_{y}$ at $t=120$ fs in Figs. 18(a) and 18(b) are the same as those in Fig. 14(b) shown on a log scale. In both cases, the peak of the field $E_{y}$ at the cluster expansion front $\left(y=r_{f}^{c l}\right)$ damps quickly with time. On the other hand, it can be seen that the spatial distribution of the field $E_{y}$ exhibiting $d E_{y} / d y<0$, i.e., the region $r_{f}^{c l} \leqslant y$, weakly depends on time, indicating that the field $E_{y}$ can be treated as a time-independent electrostatic field. We find that this tendency is also applied to the different densities $n_{a g}=0.012 n_{c}, 0.096 n_{c}$, and $0.192 n_{c}$, respectively.

In Figs. 19(a1) and 19(a2), we show the field $E_{y}$ (red solid line) obtained from the simulation at $t=93.3 \mathrm{fs}$ and the exponential function $\tilde{E}(y)$ (red dashed line) defined as Eq. (4) for the densities $n_{a g}=$ (a1) $0.024 n_{c}$ and (a2) $0.048 n_{c}$, respectively. The field $E_{y}$ is found to be well fitted by the
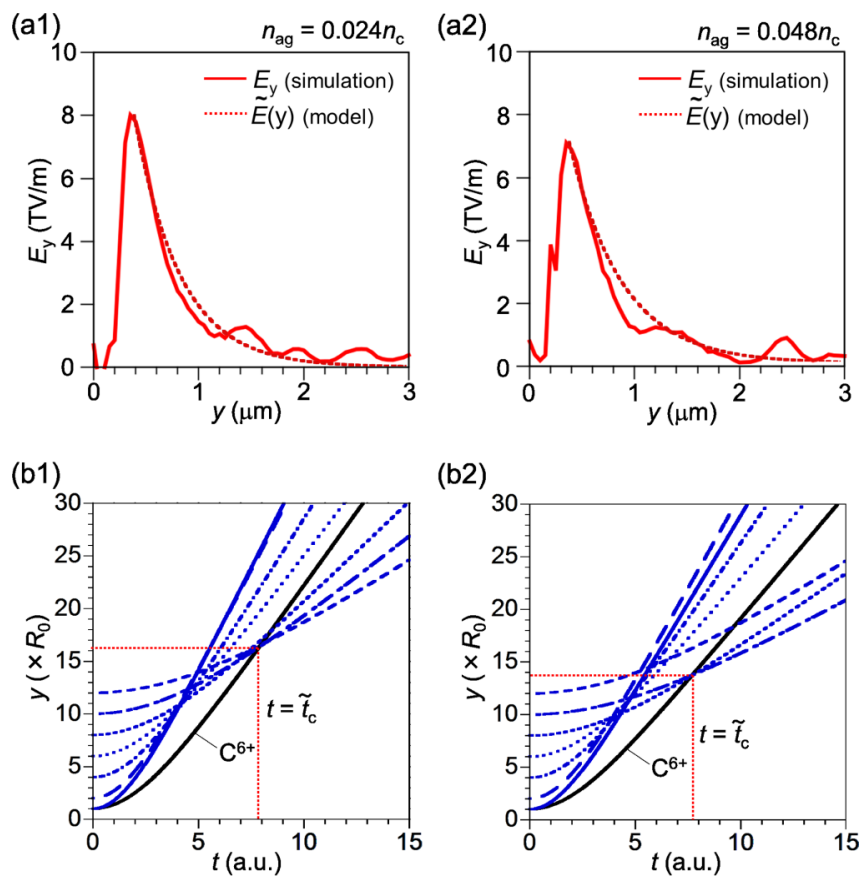

FIG. 19. (a) The spatial distribution of the field $E_{y}$ (red solid line) at $t=93.3$ fs for the density $n_{a g}=$ (a1) $0.024 n_{c}$ and (a2) $0.048 n_{c}$. The exponential function $\tilde{E}(y)$ (red dashed line) is also shown. (b) Temporal evolutions for the location of the cluster ion (black solid line), which is initially located at $y=R_{0}$, and those of the ambient gas ions (blue lines), which are initially located at $y=R_{0}, 2 R_{0}, 4 R_{0}$, $6 R_{0}, 8 R_{0}, 10 R_{0}$, or $12 R_{0}$. Note that (b1) and (b2) correspond to (a1) $0.024 n_{c}$ and (a2) $0.048 n_{c}$, respectively. The location $y$ is calculated by using the function $\tilde{E}(y)$. Red dashed line represents the time $\tilde{t}_{c}$ at which the crossing takes place.

function $\tilde{E}(y)$, as we discuss in Appendix A 2. In the following, we apply the model function $\tilde{E}(y)$ to study ion trajectories due to the characteristics of the weak time dependence of the field $E_{y}$.

Figures 19(b1) and 19(b2) show the location of a cluster ion (black solid line), which corresponds to the cluster expansion front $r_{f}^{c l}$, and also the locations of ambient gas ions (blue lines) in the $+y$ direction as a function of time $t$. Here, the cluster ion is initially located at $y=R_{0}$ and the ambient gas ions are at $y=R_{0}, 2 R_{0}, 4 R_{0}, 6 R_{0}, 8 R_{0}, 10 R_{0}$, or $12 R_{0}$, respectively.

The velocity of the cluster ion, which corresponds to the slope of the black solid line in Figs. 19(b1) and 19(b2), initially increases while finally reaching almost a constant value, which is consistent with simulation results [see black solid line in Figs. 5(a) and 12]. On the other hand, the velocities of ambient gas ions, which correspond to the slope of the blue lines in Figs. 19(b1) and 19(b2), show a similar tendency to that of the cluster ion, while the velocity of the ambient gas ion that is initially located far from the cluster front becomes smaller. Then, the ambient gas ions that are initially located at $y \geqslant 8 R_{0}$ are overtaken by the cluster ion at $t \sim \tilde{t}_{c}$ as seen in Figs. 19(b1) and 19(b2). It is found that the crossing time $t \sim \tilde{t_{c}}$ weakly depends on the densities $n_{a g}=0.024 n_{c}$ and $0.048 n_{c}$, respectively, as discussed in Sec. IV B. We have studied the trajectories of ions $\left(\mathrm{C}^{6+}\right.$ and $\left.\mathrm{H}^{+}\right)$for different 
densities $n_{a g}$ and confirmed that the crossing time $\tilde{t}_{c}$ is almost the same value, which is consistent with the result observed in Fig. 14(a).

Here, the crossing time $\tilde{t_{c}}$ observed in Figs. 19(b1) and 19(b2) can be transferred to the real value by evaluating that the slope of the black solid line in Fig. 5(a), i.e., the velocity of the cluster expansion front $\left(=2.4 \times 10^{7} \mathrm{~m} / \mathrm{s}\right)$, is equal to that in Fig. 19(b1). By using this relation, the time $t=\tilde{t_{c}} \sim 8$ at $y=16.2 R_{0} \sim 2.03 \mu \mathrm{m}\left(R_{0}=0.125 \mu \mathrm{m}\right)$ in Fig. 19(b1) corresponds to 115.6 fs. Then, under the assumption that the cluster starts to expand when the laser pulse exhibiting the half value of the laser peak intensity reaches the cluster center at $t=t_{l}=66.7 \mathrm{fs}$, the crossing time $t_{c}^{(m)}$ observed in Fig. 14(a) is estimated by $t_{c}^{(m)}=\tilde{t}_{c}+t_{l}=182.3 \mathrm{fs}$. We estimated $t_{c}^{(m)}$ for the densities $n_{a g}=0.012 n_{c}, 0.048 n_{c}, 0.096 n_{c}$, and $0.192 n_{c}$, respectively, by the procedure discussed in the above. The time $t_{c}^{(m)}$ shows the same tendency as the time $t_{c}$, i.e., almost the same value for each density $n_{a g}$ [see red dotted line in Fig. 14(a)], indicating that the procedure in deriving the time $\tilde{t_{c}}$ introduced in these two appendices is reasonable.
[1] D. Umstadter, Phys. Plasmas 8, 1774 (2001).

[2] G. A. Mourou, T. Tajima, and S. V. Bulanov, Rev. Mod. Phys. 78, 309 (2006).

[3] T. Tajima et al., Rev. Acc. Science Technol. 02, 201 (2009).

[4] A. Macchi, M. Borghesi, and M. Passoni, Rev. Mod. Phys. 85, 751 (2013).

[5] Y. Kishimoto et al., Phys. Fluids 26, 2308 (1983).

[6] E. L. Clark et al., Phys. Rev. Lett. 84, 670 (2000).

[7] R. A. Snavely, M. H. Key, S. P. Hatchett, T. E. Cowan, M. Roth, T. W. Phillips, M. A. Stoyer, E. A. Henry, T. C. Sangster, M. S. Singh, S. C. Wilks, A. MacKinnon, A. Offenberger, D. M. Pennington, K. Yasuike, A. B. Langdon, B. F. Lasinski, J. Johnson, M. D. Perry, and E. M. Campbell, Phys. Rev. Lett. 85, 2945 (2000).

[8] S. C. Wilks et al., Phys. Plasmas 8, 542 (2001).

[9] L. O. Silva, M. Marti, J. R. Davies, R. A. Fonseca, C. Ren, F. Tsung, and W. B. Mori, Phys. Rev. Lett. 92, 015002 (2004).

[10] A. Macchi, F. Cattani, T. V. Liseykina, and F. Cornolti, Phys. Rev. Lett. 94, 165003 (2005).

[11] D. Haberberger et al., Nat. Phys. 8, 95 (2012).

[12] F. Fiuza et al., Phys. Plasmas 20, 056304 (2013).

[13] L. Romagnani et al., Phys. Rev. Lett. 101, 025004 (2008).

[14] Y. Kuramitsu et al., Phys. Rev. Lett. 106, 175002 (2011).

[15] N. L. Kugland et al., Nat. Phys. 8, 809 (2012).

[16] H. Ahmed et al., Phys. Rev. Lett. 110, 205001 (2013).

[17] J. Meinecke et al., Nat. Phys. 10, 520 (2014).

[18] B. Albertazzi et al., Science 346, 325 (2014).

[19] C. M. Huntington et al., Nat. Phys. 11, 173 (2015).

[20] N. G. Gotts et al., J. Chem. Phys. 96, 408 (1992).

[21] J. Purnell et al., Chem. Phys. Lett. 229, 333 (1994).

[22] M. Lezius, S. Dobosz, D. Normand, and M. Schmidt, Phys. Rev. Lett. 80, 261 (1998).

[23] T. Ditmire, R. A. Smith, J. W. G. Tisch, and M. H. R. Hutchinson, Phys. Rev. Lett. 78, 3121 (1997).

[24] T. Ditmire et al., Nature (London) 386, 54 (1997).

[25] T. Fennel, K. H. Meiwes-Broer, J. Tiggesbaumker, P. G. Reinhard, P. M. Dinh, and E. Suraud, Rev. Mod. Phys. 82, 1793 (2010).
[26] Y. Kishimoto et al., Phys. Plasmas 9, 589 (2002).

[27] T. Ditmire et al., Nature (London) 398, 489 (1999).

[28] Y. Fukuda et al., JETP Lett. 78, 115 (2003).

[29] M. Uesaka, Femtosecond Beam Science (Imperial College Press, 2005), p. 165.

[30] T. Nakamura, Y. Fukuda, and Y. Kishimoto, Phys. Rev. A 80, 053202 (2009).

[31] R. Rajeev et al., Nat. Phys. 9, 185 (2013).

[32] R. Matsui, Y. Fukuda, and Y. Kishimoto, Phys. Rev. Lett. 122, 014804 (2019).

[33] A. S. Boldarev et al., Rev. Sci. Instrum. 77, 083112 (2006).

[34] S. Jinno et al., Opt. Express 21, 20656 (2013).

[35] D. G. Jang et al., Appl. Phys. Lett. 105, 021906 (2014).

[36] T. Ditmire et al., Astrophys. J. Suppl. Ser. 127, 299 (2000).

[37] D. R. Symes et al., Nucl. Instrum. Methods Phys. Res. A 653, 186 (2011).

[38] A. Marocchino et al., Appl. Phys. Lett. 112, 264104 (2018).

[39] F. P. A. Vogt et al., Nat. Astron. 2, 465 (2018).

[40] N. Iwata et al., Phys. Plasmas 23, 063115 (2016).

[41] Y. Kishimoto and T. Masaki, J. Plasma. Phys. 72, 971 (2006).

[42] S. Jinno et al., Appl. Phys. Lett. 102, 164103 (2013).

[43] M. Kanasaki et al., Plasma Phys. Controlled Fusion 58, 034013 (2016)

[44] M. V. Ammosov, N. B. Delone, and V. P. Krainov, Sov. Phys. JETP 64, 1191 (1986).

[45] W. L. Zhang et al., Phys. Plasmas 23, 073118 (2016).

[46] Y. Kishimoto et al., J. Phys. Soc. Jpn. 59, 118 (1990).

[47] K. Imadera et al., J. Comput. Phys. 228, 8919 (2009).

[48] I. B. Bernstein, J. M. Greene, and M. D. Kruskal, Phys. Rev. 108, 546 (1957).

[49] R. C. Davidson, Methods in Nonlinear Plasma Theory (Academic Press, New York, 1972), p. 75.

[50] H. Matsumoto et al., Geophys. Res. Lett. 21, 2915 (1994).

[51] L. Muschietti et al., Geophys. Res. Lett. 26, 1093 (1999).

[52] Y. Kishimoto, T. Watanabe, H. Hojo, and K. Nishikawa, J. Phys. Soc. Jpn. 51, 2304 (1982). 